\title{
The Structure of Spatial Receptive Fields of Neurons in Primary Auditory Cortex of the Cat
}

\author{
John F. Brugge, Richard A. Reale, and Joseph E. Hind \\ Department of Neurophysiology and Waisman Center, University of Wisconsin, Madison, Wisconsin 53706
}

Transient broad-band stimuli that mimic in their spectrum and time waveform sounds arriving from a speaker in free space were delivered to the tympanic membranes of barbiturized cats via sealed and calibrated earphones. The full array of such signals constitutes a virtual acoustic space (VAS). The extracellular response to a single stimulus at each VAS direction, consisting of one or a few precisely time-locked spikes, was recorded from neurons in primary auditory cortex. Effective sound directions form a virtual space receptive field (VSRF). Near threshold, most VSRFs were confined to one quadrant of acoustic space and were located on or near the acoustic axis. Generally, VSRFs expanded monotonically with increases in stimulus intensity, with some occupying essentially all of the acoustic space. The VSRF was not homogeneous with respect to spike timing or firing strength. Typically, onset latency varied by as much as $4-5 \mathrm{msec}$ across the VSRF. A substantial proportion of recorded cells exhibited a gradient of first-spike latency within the VSRF. Shortest latencies occupied a core of the VSRF, on or near the acoustic axis, with longer latency being represented progressively at directions more distant from the core. Remaining cells had VSRFs that exhibited no such gradient. The distribution of firing probability was mapped in those experiments in which multiple trials were carried out at each direction. For some cells there was a positive correlation between latency and firing probability.

Key words: Al; primary auditory cortex; sound localization; directional hearing; spatial receptive fields; virtual acoustic space; spatial hearing
Primary auditory cortex (AI) plays a central role in directional hearing. Neurons of AI have been shown under dichotic conditions to be sensitive to acoustical cues used by listeners in localizing a static sound source and detecting sound motion. Under free-field conditions, AI cells exhibit spatial tuning and motion sensitivity. Presumably, it is the interruption of the output of these neurons that contributes to directional hearing deficits resulting from AI lesions (for review, see Brugge and Reale, 1985; Clarey et al., 1992; Phillips, 1995).

At moderate sound intensity, spatial receptive fields of AI neurons are typically large, often occupying a quadrant or more of acoustic space (Middlebrooks and Pettigrew, 1981; Brugge et al., 1994, 1996). This finding questions how broadly tuned elements contribute to auditory spatial acuity while they integrate information about sounds moving with respect to the head and ears as well as static sounds arriving at the same or different times from wide areas of acoustic space. Dichotic studies of primary auditory cortical neurons have shown that discharge timing and strength change systematically with changes in the major localization cues of interaural intensity (IID) or interaural time (ITD) differences (Brugge et al., 1969; Phillips and Irvine, 1981; Reale and Brugge, 1990). Changing the direction of a broad-band sound source in space produces changes in ITD and IID, as well as changes in

\footnotetext{
Received Jan. 25, 1996; revised April 24, 1996; accepted April 26, 1996.

This work was supported by National Institutes of Health Grants DC00116, DC00398, and HD03352. We acknowledge the participation of Joseph C. K. Chan, Paul W. F. Poon, Alan D. Musicant, and Mark Zrull in many of the experiments related to virtual space receptive fields that are presented here. Jiashu Chen and Zenyang Wu played major roles in the development of the FIR filter approach. Ravi Kochhar was responsible for developing the software that implemented virtual acoustic space. Richard Olson, Dan Yee, and Bruce Anderson were responsible for the instrumentation.

Correspondence should be addressed to John F. Brugge, 627 Waisman Center, University of Wisconsin, Madison, WI 53705.

Copyright (C) 1996 Society for Neuroscience $0270-6474 / 96 / 164420-18 \$ 05.00 / 0$
}

spectrum attributable to the transfer characteristics of the head and external ear. It might be expected, therefore, that response properties would vary with sound direction, as they do with changes in interaural localization cues studied in isolation. Responses of AI neurons to tones or noise in the free field already indicate that functional gradients based on response magnitude may exist along the azimuthal dimension within a spatial receptive field (Imig et al., 1990; Rajan et al., 1990a,b; Samson et al., 1994).

Systematic and detailed study of auditory spatial receptive field properties has been limited by technical difficulties associated with generating signals and controlling their direction over wide expanses of acoustic space at high spatial resolution. We have overcome these difficulties by developing techniques to deliver to the tympanic membranes over calibrated earphones signals that mimic in their spectrum and time waveform sounds arriving from a speaker in free space (Brugge et al., 1994, 1996; Chen et al., 1994, 1995; Reale et al., 1996). The full array of such signals constitutes a virtual acoustic space (VAS). Using this approach, we found previously that AI neurons of cat are sensitive to the direction of an impulsive sound and that the directions in VAS that are effective in exciting a cortical neuron aggregate to form a virtual space receptive field (VSRF). Our initial studies focused on the methods for generating a VAS, the classes of VSRFs found in a sample of AI neurons, and binaural interactions that tend to shape a VSRF (Brugge et al., 1994, 1996; Reale et al., 1996). We now turn attention to the detailed structure of the spatial receptive field of AI neurons. We first describe the spatial tuning properties of AI neurons over a range of stimulus intensity and then show that within the spatial receptive field there may be an orderly representation of response timing or response magnitude or both.

\section{MATERIALS AND METHODS}

The methods used in this study were the same as those reported elsewhere (Chan et al., 1993; Brugge et al., 1994, 1996; Chen et al., 1994, 1995; Reale et al., 1996). Briefly, barbiturate-anesthetized cats were fitted 
with hollow ear pieces sealed into the external ear canals through which VAS signals were delivered via a calibrated sound-delivery system. The acoustic drivers were the same type (Radio Shack Super Tweeter, model 40-1310A) used to generate the original free-field signals (Musicant et al., 1990) but were modified for our insert sound system (Chan et al., 1993). For each experiment, the sound-delivery system was calibrated in situ, and the amplitude and phase of a pure tone, at frequencies between $156 \mathrm{~Hz}$ and $40 \mathrm{kHz}$, were stored by computer. These calibration data, together with the impulse response of the delivery system (Chen et al., 1994), were used to correct the VAS signals for the spectrum-altering effects of the sound-delivery system.

A VAS was derived from sound-pressure measurements made at the eardrums of cats as part of an earlier study (Musicant et al., 1990). In that study, a rectangular pulse (10 $\mu$ s duration) was used to excite a free-field speaker, the direction of which was varied in a spherical coordinate system, and a set of free-field to eardrum transfer functions (FETFs) was obtained. The FETF expresses, for a given source-direction over a specified range of frequencies, the transformations of amplitude and phase that occur from sound pressure measured in the free field in the absence of a subject to the pressure measured at the eardrum when the subject is introduced into the sound field. Although for the same sound-source direction there can be significant differences among cats in the absolute values of the spectral transformation, across animals the general patterns of location-dependent spectral features are similar (Musicant et al., 1990; Rice et al., 1992). We have shown elsewhere (Reale et al., 1996) that despite the differences in VAS from one cat to the next, the VSRFs obtained using transfer functions from different cats can be remarkably similar.

In the present study, stimuli derived from the same transient signals were used, and sound-source directions were referred to the same spherical coordinate system centered on the cat's interaural plane that covered $360^{\circ}$ in azimuth and $126^{\circ}$ in elevation. Measurements were not made at elevations below $-36^{\circ}$ (Musicant et al., 1990) and thus were not represented in our VAS. Typically, VAS was represented by an array of 1650 waveform pairs spaced at 4.5 or $9^{\circ}$ intervals; at each direction the pair of signals, appropriate for the left and right ears, was simulated digitally. Signal intensity could be varied over a range of $127 \mathrm{~dB}$ from its maximal level (full-scale output from a 12-bit D/A converter). In this study, intensity is expressed in decibels of attenuation (dB ATTN) from this maximum. Psychophysical studies using similar signal-processing methods have shown that human listeners perform localization tasks as well under these earphone-listening conditions as they do under free-field conditions (Wightman et al., 1987; Wightman and Kistler, 1989a,b).

Single neurons (248) were recorded extracellularly in the highfrequency region of the left auditory cortex of 28 cats, and their responsiveness to virtual-space stimuli was tested subsequently. As used here, the terms contralateral and ipsilateral refer to the cerebral hemispheric location of a recorded cell. Quantitative data were obtained from 25 of these experiments. Typically, neurons were isolated while search stimuli tone-bursts that varied in frequency and sound pressure level (SPL) were used. Most neurons (208) in our sample population that responded to tonal search stimuli also responded securely and consistently to all or a subset of the virtual-space signals. Characteristic frequency $(\mathrm{CF})$, the frequency to which a neuron responds at the lowest SPL, was ascertained from neurons or neuronal clusters in a sufficient number of electrode penetrations to verify that the single cells studied were within the boundary of the tonotopic map of AI (Merzenich et al., 1975; Reale and Imig, 1980). Binaural interactions were usually evaluated qualitatively using CF tone-bursts and were classified using the following simple scheme: EI interaction is one in which a neuron received excitatory input from one ear and inhibitory input from the other, with a resulting binaural inhibition; EE interaction refers to excitatory input from each ear, and a facilitatory or summative binaural response; and PB interaction is one in which stimulation of both ears within a narrow range of IID was required to excite the cell. Although qualitative judgments of binaural interaction gave on-line guidance to our experiments, time restraints generally precluded quantitative response area measurements. Thus, we have simply indicated in the text or figure legends the binaural classes for each of the neurons presented in figures.

One hundred fifty-nine cells were studied long enough to obtain at least one complete VSRF, usually at intensities within 10-30 dB of threshold. CFs of these neurons ranged from $\sim 9 \mathrm{kHz}$ to $40 \mathrm{kHz}$. The effects of changing intensity on the VSRF were studied for 87 of those 159 cells. Thirty cells were studied at two intensities, 29 at three intensities, 16 at four intensities, 8 at five intensities, and 4 at six intensities. These experiments involved changes in intensity at both ears in steps of 5,10, or $20 \mathrm{~dB}$. Intensity was varied over a range of $55 \mathrm{~dB}$, usually starting $\sim 10-30$ $\mathrm{dB}$ above threshold. Threshold was taken as the minimal intensity necessary to evoke a consistent discharge from the cell at or near its most sensitive VAS direction.

Before quantitative data were collected, responses to VAS stimulation were assessed qualitatively. VSRFs were then derived from responses to single presentations of VAS stimuli delivered dichotically at each direction in random order. Approximately $15 \mathrm{~min}$ was required to obtain a VSRF representing the responses to a single stimulus at each of 1650 directions. In several experiments we also studied responses to 5-15 repetitions of a VAS stimulus at each direction; the stimulus repetition rate was $2 / \mathrm{sec}$. Because of time limitations, the extent of the VSRF mapped with multiple stimulus trials was usually confined to approximately one quadrant of VAS. As a rule, VSRFs were very stable over the several hours during which we studied a neuron (Brugge et al., 1994).

We applied three metrics to the VSRF (also see Brugge et al., 1996). For each VSRF we computed a laterality index (LI), which is a simple measure that compares the number of effective directions located contralateral (right) and ipsilateral (left) of the sagittal midline [LI = $(\mathrm{C}-\mathrm{I}) /(\mathrm{C}+\mathrm{I})]$. This metric can range from -1 , when the VSRF is completely confined to the ipsilateral hemifield, to +1 , when the VSRF is similarly restricted to the contralateral hemifield; a value of 0 implies that the sagittal midline bisects the VSRF.

The spherical area (SA) was used to estimate the spatial extent of the VSRF. The SA of the VSRF (expressed in spherical degrees) was computed by linear interpolation between neighboring sampled directions. A spherical degree is that portion of a sphere enclosed by a spherical triangle with two sides each having arcs of $90^{\circ}$, and the third side having an arc of $1^{\circ}$. Thus, the area of a sphere is 720 spherical degrees. Because the VAS procedure we used was limited to sound-source directions not more than $36^{\circ}$ below the interaural plane, the largest VSRF possible would have an area of $\sim 615$ spherical degrees.

The spherical median (SM) was used to express the central tendency of the spatial distribution of VAS directions effective in discharging the cell. The SM is that particular direction for which the average value of the angles made with receptive field directions is minimized. The spherical mean direction of a VSRF is a useful metric when the spatial pattern appears unimodal and rotationally symmetric about some direction. Because most VSRFs in our sample exhibited marked asymmetry, a statistic analogous to the median of a sample of linear data is preferred, because it is less influenced by the extreme values in the sample set. In those cases in which the VSRF is symmetric about some direction, the spherical mean and median directions are the same (Fisher et al., 1987). Because we did not have stimuli representing directions below $-36^{\circ}$ elevation, there is a bias in our estimates of the median elevation, when VSRFs are large and capable of encroaching on that region of virtual space. All VSRFs were subject to the same bias, which was not corrected.

\section{RESULTS}

For most AI neurons studied (76\%), the VSRF obtained $\sim 10-30$ $\mathrm{dB}$ above threshold was confined largely to a quadrant of acoustic space in front of the animal. These could be placed in one of three classes (Brugge et al., 1994). Approximately 69\% of the VSRFs were centered in either the contralateral or the ipsilateral frontal quadrant of VAS, with the greatest number being contralateral. These were classified as "contralateral" or "ipsilateral" or collectively as "hemifield" neurons. A smaller proportion of AI cells (7\%) responded to sounds arising from directions centered on or near the frontal midline. These were classified as "frontal" neurons. The remainder of the neurons studied were placed in the "omnidirectional" or "complex" category.

For the great majority $(\sim 90 \%)$ of the 87 cells drawn from this population and studied at more than one stimulus level, there was an increase in the area of the receptive field with increases in intensity. Near threshold, the size and shape of the VSRF is governed mainly by pinna transformations (Middlebrooks and Pettigrew, 1981), whereas at moderate to high intensities the VSRF depends in large part on both the pinna transformations and the type and strength of binaural interactions (Brugge et al., 1994). Thus, the classification of a neuron based on VSRF prop- 
erties near threshold does not necessarily predict the receptive field behavior of the cell at higher stimulus levels. Because omnidirectional and complex cells made up a relatively small proportion of the total, and because spatial tuning of omnidirectional cells changes little with intensity, we concentrated on studying cells classified as contralateral, ipsilateral, or frontal. Broad auditory spatial tuning is not attributable to general anesthesia (Benson et al., 1981) nor is it confined to AI of the cat (Knudsen et al., 1977; Benson et al., 1981; Semple et al., 1983; Moore et al., 1984a,b).

The VSRFs are plotted on the same spherical coordinate system used to obtain the FETFs from which the VAS was derived (Musicant et al., 1990; Brugge et al., 1994). Figure 1 is an example of the spheres, shown bisected into front and rear acoustic hemifields and hinged at a single locus at coordinates $+90^{\circ}$ azimuth and $0^{\circ}$ elevation. Thus, the front acoustic hemifield represents the interior of the imaginary sphere as seen from the cat's point of view; the rear hemifield represents the interior of the sphere behind the animal. In this bisected view, contralateral acoustic space occupies the right-hand quadrant of the front hemifield and the adjacent left-hand quadrant of the rear hemifield. Ipsilateral space occupies the remaining two nonadjacent quadrants. Each direction that was effective in evoking at least one spike is denoted by a black area centered at that sampled locus in VAS. Tested but ineffective directions are shown only on the top-most VSRF by small black dots (in subsequent figures the dots are not included for reasons of clarity). The SA and LI associated with each VSRF are shown to the left.

\section{Spatial tuning of Al neurons}

For the two neurons illustrated in Figure 1, VSRFs obtained near threshold were found to lie mainly in either the contralateral frontal quadrant of VAS in the case of one cell (left column, $75 \mathrm{~dB}$ ATTN) or the ipsilateral quadrant in the case of the other (right column, $65 \mathrm{~dB}$ ATTN). Both cells exhibited hemifield VSRFs for intensities within $20 \mathrm{~dB}$ of threshold. When intensity was increased further, the VSRF expanded and eventually came to represent essentially all of the acoustic space surrounding the cat. Quantitatively, this is reflected in the systematic decrease in the LI and the systematic increase in the SA. We refer to VSRFs with this expansive property as being "unbounded."

For other hemifield neurons, the VSRF expanded with increases in intensity, but that expansion was confined largely to stimulus directions in either contralateral or ipsilateral space. Figure 2 illustrates VSRFs from two such neurons obtained over a range of $30 \mathrm{~dB}$. In both cases, the VSRF at any tested intensity consisted mainly of directions in contralateral space. For the neuron shown at the left, expansion of the VSRF associated with increasing intensity occurred by recruiting new effective directions, predominately in the rear contralateral quadrant but also at high and low elevations in the contralateral frontal quadrant. This change in spatial pattern with increasing intensity is mirrored in the changes in both the SA and the LI. We refer to such VSRFs as being "bounded," a term originally used by Semple et al. (1983) to describe similar patterns of spatial receptive fields of inferior colliculus neurons. The terms "bounded" and "unbounded" were also used by Imig et al. (1990) to describe the width of azimuthal functions derived from AI responses to noise bursts in the free field.

Whether bounded or unbounded, the spatial configuration of a VSRF took different forms in different cells. Unlike the marked juxtaposition of effective directions that make up the VSRFs exhibited by the neuron shown to the left, the VSRFs shown in the right-hand column of Figure 2 appear "fractured," in the sense that the effective directions were often separated from one another by ineffective directions. Near threshold (at $45 \mathrm{~dB}$ ATTN) the effective directions were confined to, but scattered throughout, the contralateral acoustic hemifield. Raising the intensity by $10 \mathrm{~dB}$ increased the number of effective directions within the contralateral space, thereby creating a more densely packed VSRF, with a slight expansion of the VSRF into the lower rear contralateral quadrant. The laterality of the VSRF, however, was affected little by change in intensity, as reflected in the high and relatively constant LI. Instead, the spatial distribution of responses within the VSRF changed, reverting to the fractured pattern exhibited near threshold. Thus, the SA becomes a nonmonotonic function of intensity. This nonmonotonic and fractured relationship with intensity is not the result of changes over time in the general excitability of the cell, because we have observed it on repeated runs at different times during an experiment. In fact, we find that VSRFs are quite stable in their form and structure over relatively long periods (Brugge et al., 1994). Rather, we suspect that in such cells the influence of direction on the discharge cannot be accounted for simply by static directional changes in acoustic features of the stimulus, including IID and ITD. All classes of cells had fractured and nonfractured VSRFs regardless of whether the VSRFs were bounded or unbounded. On the basis of qualitative examination of VSRFs in our entire database, we have concluded that any measure of the contiguity of effective directions within a VSRF would form a continuum. Approximately one third of recorded cells exhibited the degree of fracture seen in Figure 2. Other examples of even more complex fractured VSRFs are presented later in the paper.

Frontal VSRFs may be expressed when stimulation of each ear alone is excitatory and the binaural interaction is facilitatory (EE) or when excitation requires stimulation of the two ears together (PB). Figure 3 illustrates changes in VSRFs from two frontal neurons whose binaural interactions were classified as EE under dichotic tonal conditions. For both neurons illustrated here, at near-threshold intensity the VSRF had a major aggregation of effective directions in each frontal quadrant of VAS, which were distributed around a parallel of $\sim 18^{\circ}$ of elevation and joined at the midline. Although we do not have monaural VSRF data for this cell, the clusters of effective directions in each frontal hemifield likely represent the independent monaural excitatory responses (Brugge et al., 1994). For the cell shown on the left, the VSRF was fractured and biased toward contralateral space (as reflected in a positive LI). For the cell shown on the right, the VSRF was densely packed and nearly bisected by the vertical meridian $(\mathrm{LI} \sim 0)$. When stimulus intensity was raised, both neurons became omnidirectional and SA increased in a monotonic fashion, with the spread of effective directions remaining more or less symmetric around the vertical meridian.

VSRFs from two frontal neurons whose binaural interactions were classed as PB are illustrated in Figure 4. They each showed a single aggregation of effective directions on or slightly to the right or left of the midline (LI is slightly positive or negative), and as the stimulus level was raised, they became omnidirectional (SA grows monotonically). Samson et al. (1994) included in their "binaurally directional" (BD) category PB neurons with azimuthal functions having similar behavior. VSRFs derived from these cells also may exhibit fracturing. As a rule, frontal neurons were unbounded as the stimulus level was raised.

Results from 14 selected neurons with properties similar to 


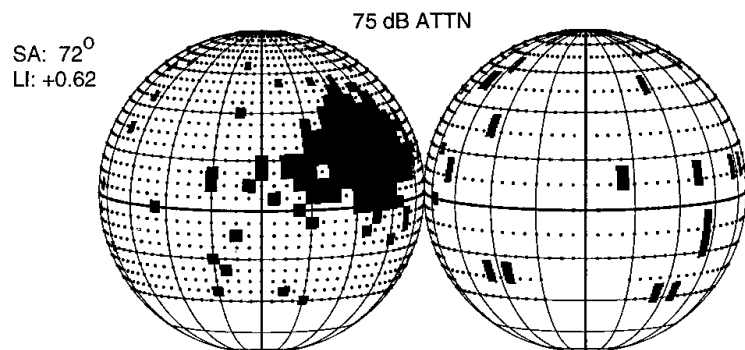

$70 \mathrm{~dB}$ ATTN
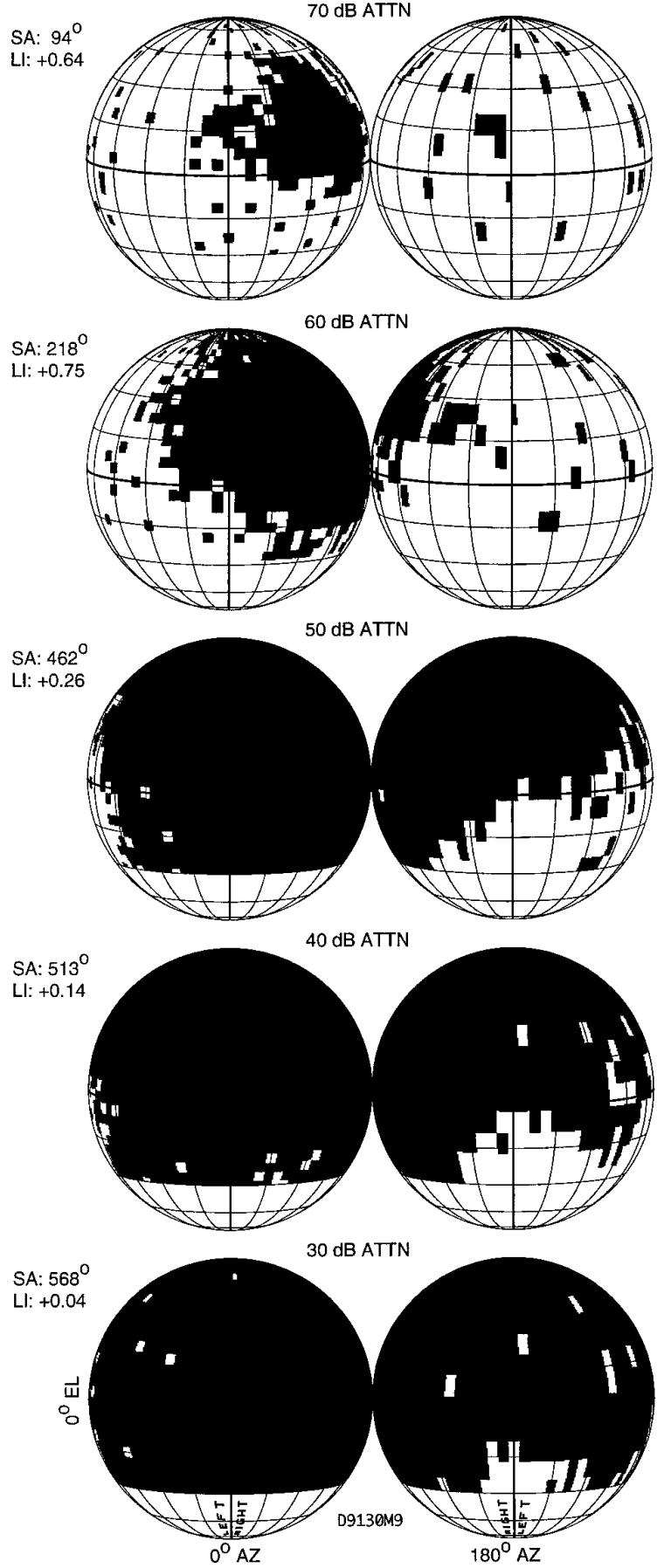

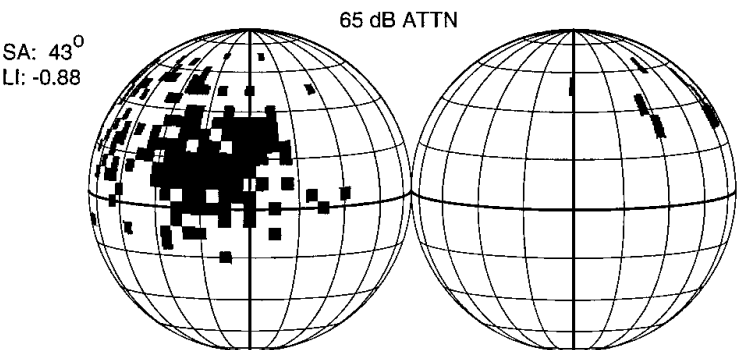

$60 \mathrm{~dB}$ ATTN

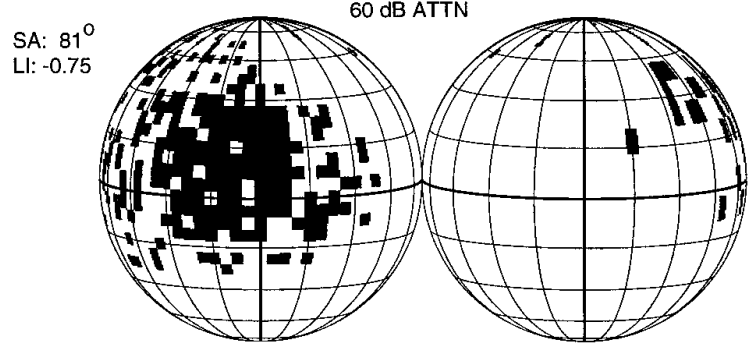

$50 \mathrm{~dB}$ ATTN
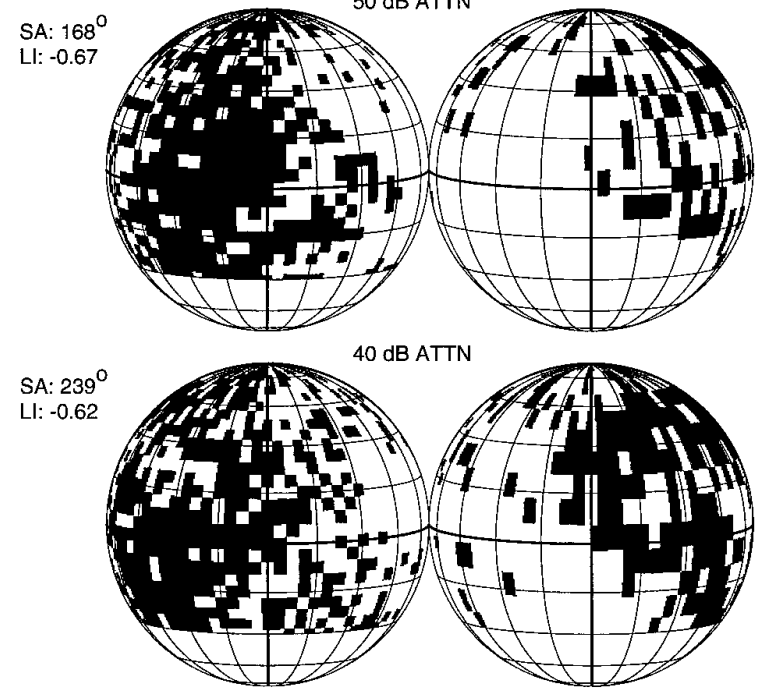

$20 \mathrm{~dB}$ ATTN
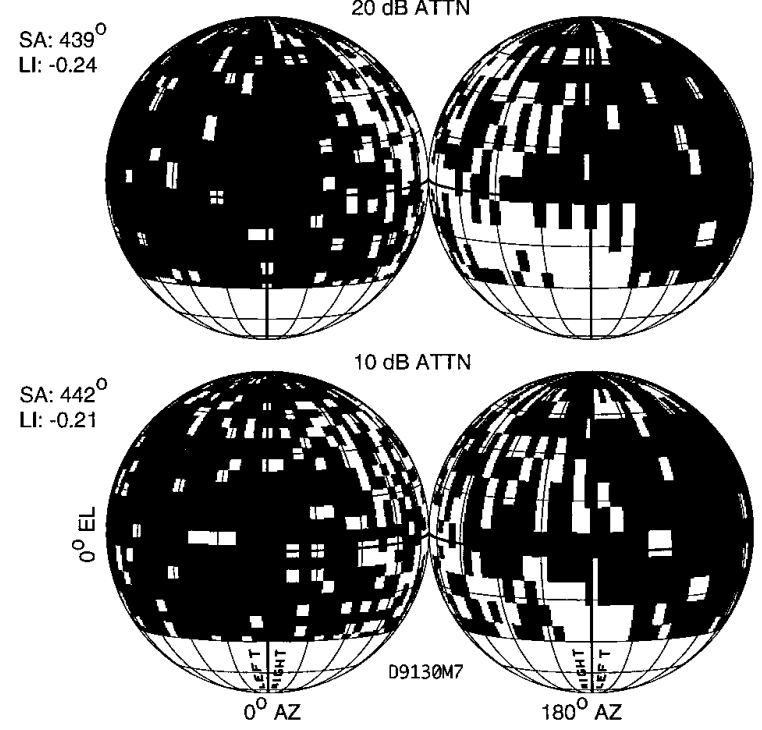

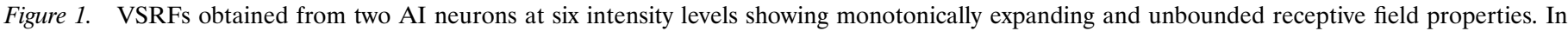

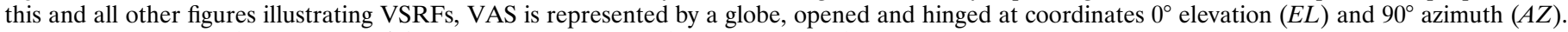

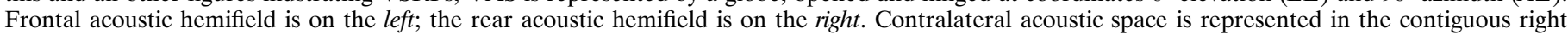

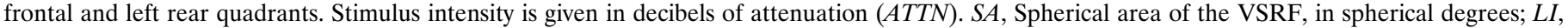
laterality index. CF-tone binaural interaction: D9130M9, EI; D9130M7, EE. See text for further details. 

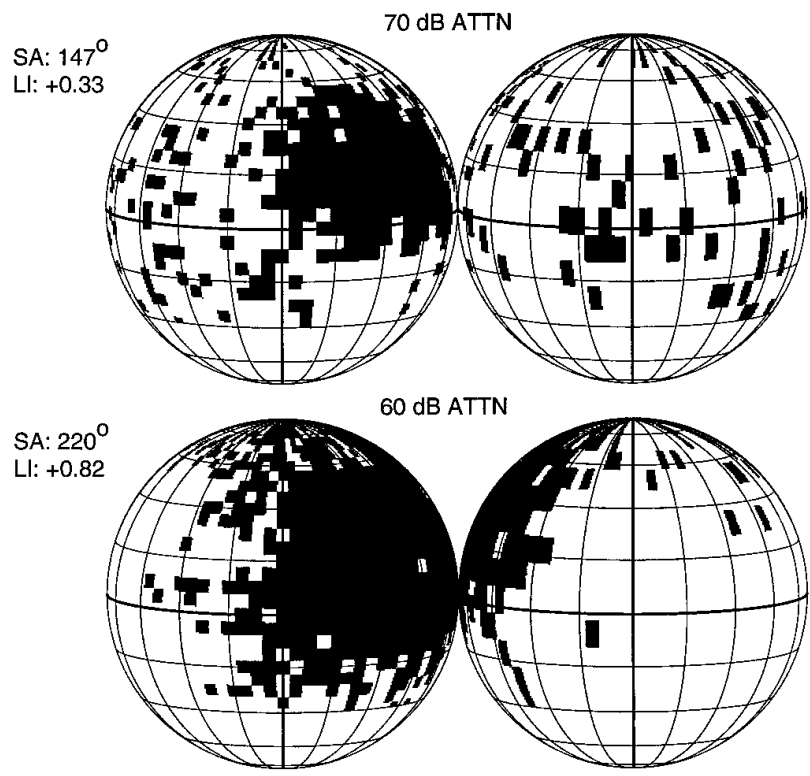

$40 \mathrm{~dB}$ ATTN

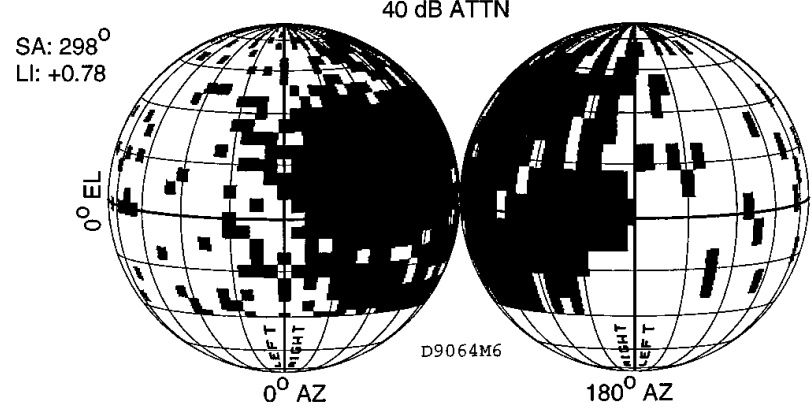

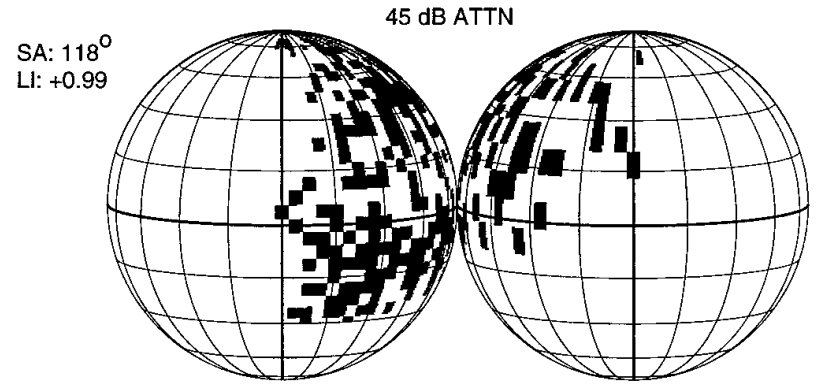

$35 \mathrm{~dB}$ ATTN

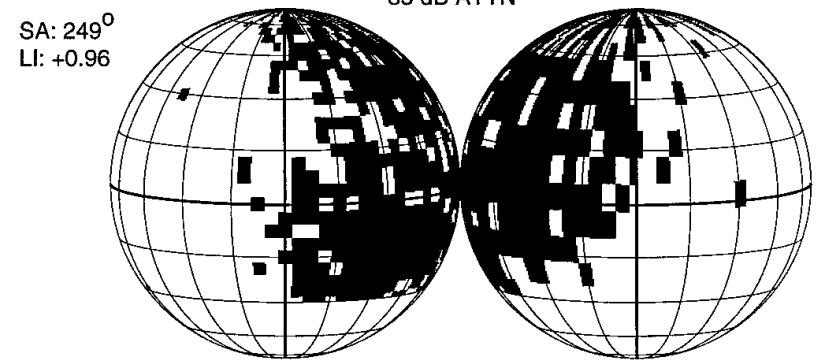

$25 \mathrm{~dB}$ ATTN

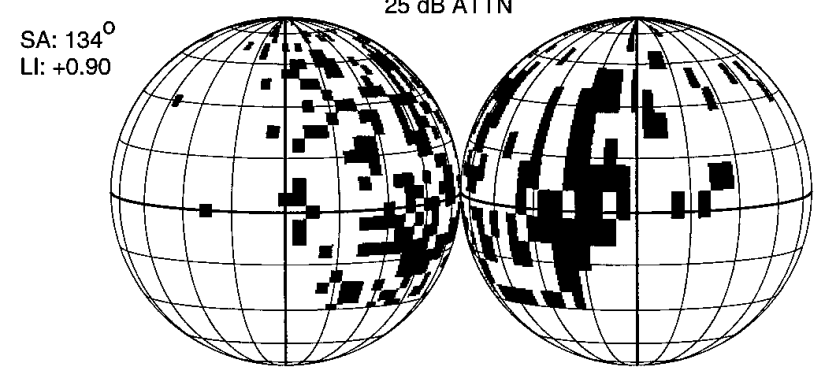

$15 \mathrm{~dB}$ ATTN

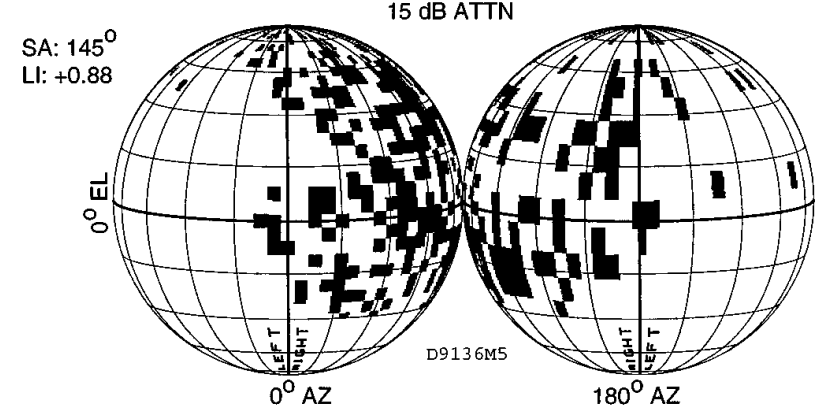

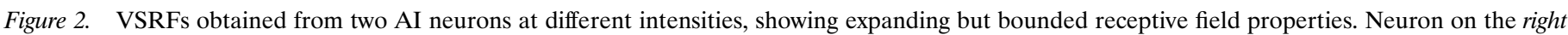
exhibits a "fractured" pattern. CF-tone binaural interaction: D9064M6, EI; D9136M5, EI. See text and legend to Figure 1 for further details.

those illustrated in Figures 1-4, and for which we have data at three or more intensities, are summarized in Figure 5. Here LI and SA are plotted as a function of stimulus level for each of the groups of neurons illustrated above. Neurons classified as "hemifield" (contralateral or ipsilateral) having VSRFs that were unbounded showed high (both positive and negative) LI (Fig. 5A) and small SA (Fig. $5 B$ ) at low intensity, but as stimulus intensity was raised, LI decreased toward zero and the SA increased monotonically. The shallow increase in SA exhibited by one cell (squares) reflects the fractured nature of those VSRFs. VSRFs of bounded neurons were constrained to contralateral or ipsilateral space as seen by the relatively high LI (Fig. 5C) and relatively low SA (Fig. 5D) maintained by these cells in the face of increasing stimulus intensity. Frontal neurons also exhibited VSRFs that grew in area (Fig. $5 F$ ), as shown by the monotonically increasing SA. Again, one neuron illustrated here (squares) exhibited a substantially fractured VSRF. The VSRFs also remained centered near the midline as the stimulus level was raised, which is reflected in the relatively low and constant LI (Fig. 5E).

As we reported previously, $\sim 8 \%$ of the neurons in our total sample exhibited a "complex" VSRF (Brugge et al., 1994). For neurons in this complex population, increases in intensity produced changes in the spatial pattern of effective directions that seemed more complicated than those seen in the relatively simple bounded and unbounded VSRFs described above. Data from two such neurons, obtained over a range of intensity, are illustrated in Figure 6. In both cases, an aggregate of directions that was effective at the lowest intensity tested became ineffective at a higher intensity. For the neurons illustrated on the right of the figure, the VSRF obtained at $50 \mathrm{~dB}$ ATTN showed an aggregate that extended from $0^{\circ}$ to $\sim 18^{\circ}$ in elevation and from the midline to $\sim 36^{\circ}$ in azimuth. At the higher intensity of $30 \mathrm{~dB}$ ATTN, this region was virtually devoid of activity, although at still higher intensities a fractured pattern developed in the region. Similarly, 

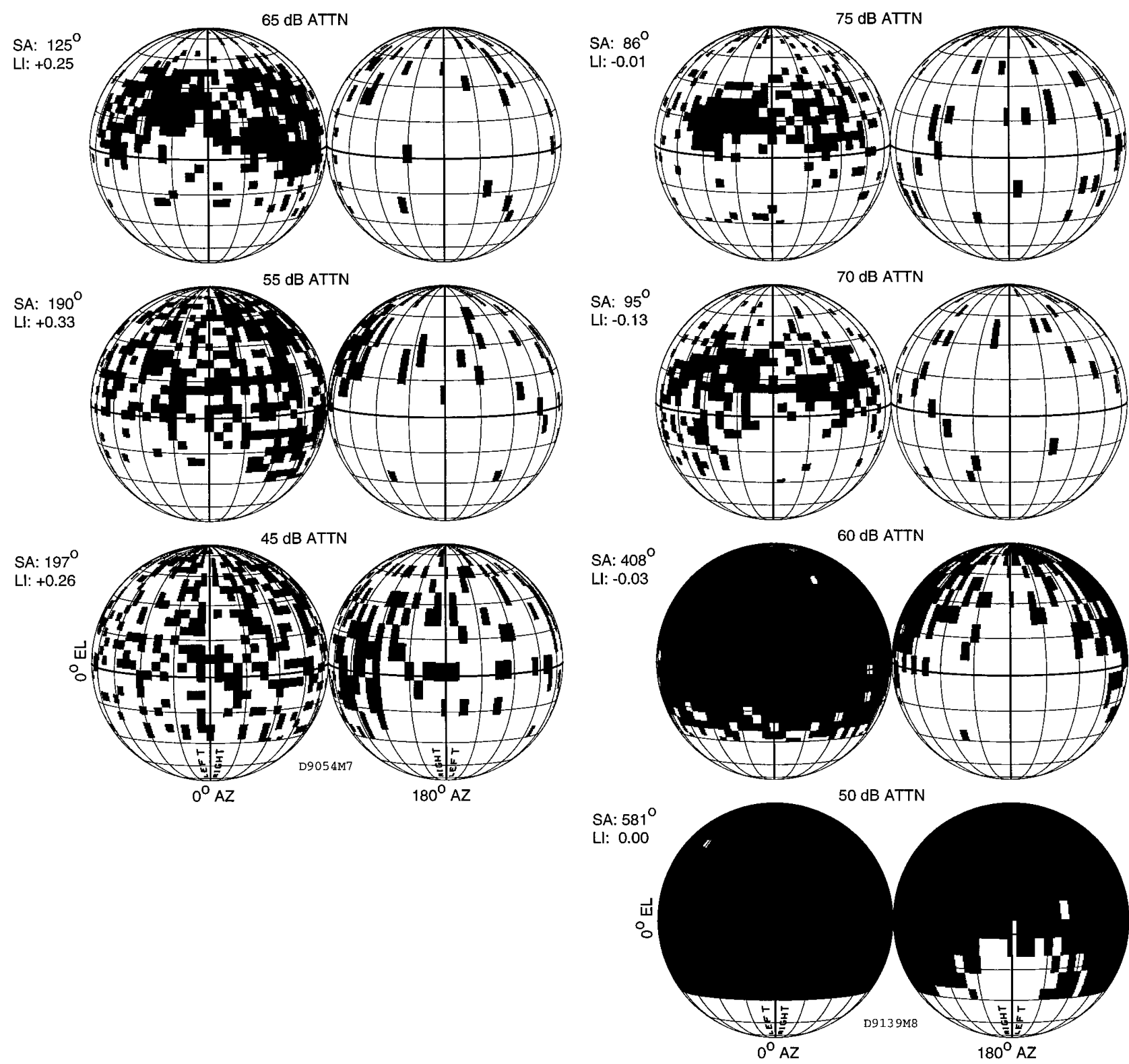

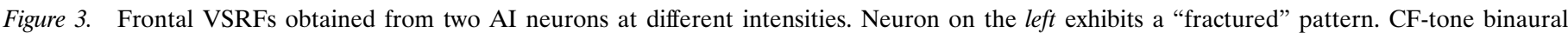
interaction: D9054M7, EE; D9139M8, EE. See text and legend to Figure 1 for further details.

for the neuron illustrated in the left column, several regions of effective directions at 80 or $75 \mathrm{~dB}$ ATTN were found subsequently to be ineffective at the higher intensities of 65 and $55 \mathrm{~dB}$ ATTN. In this case, directions that were effective at low intensities became ineffective at high, and remained so at even higher intensities, resulting in a VSRF with both a lower and an upper threshold. The resulting dynamic range of this cell was $\sim 30 \mathrm{~dB}$. We have studied one other cell with an apparent dynamic range of only 20 dB. "Omnidirectional" cells remained omnidirectional over the range of intensities studied and hence are not illustrated here.

\section{Relationship of the VSRF to the acoustic axis}

It has been observed that spatial receptive fields in the contralateral or ipsilateral acoustic space, such as those illustrated in Figure 1, are centered near or around the acoustic axis (also see Middlebrooks and Pettigrew, 1981). The acoustic axis marks the direction at which the transformation of sound pressure by the pinna achieves maximum amplification for a given frequency. We tested directly whether such a relationship existed by first estimating the center of the VSRF for hemispheric neurons using the SM (see Materials and Methods). The VSRFs chosen were those obtained within 10-30 dB of threshold; they had relatively focal distributions of responsive loci, similar to that illustrated in Figure 1. We then compared the spatial distribution of SMs with the spatial distribution of the acoustic axes, obtained from the VAS, at frequencies between $\sim 9 \mathrm{kHz}$ and $40 \mathrm{kHz}$, which covers the range of CFs of neurons in this sample population.

Figure $7 A$ shows the spatial locations of SMs (open circles) of 65 VSRFs and of the acoustic axes (solid circles), plotted on the same coordinate system used to display the VSRFs. Fifty-four of the SMs plotted are from VSRFs in the contralateral quadrant; the remaining 11 are from VSRFs in ipsilateral space. The acoustic axes are represented mirror-symmetrically around the midline. 

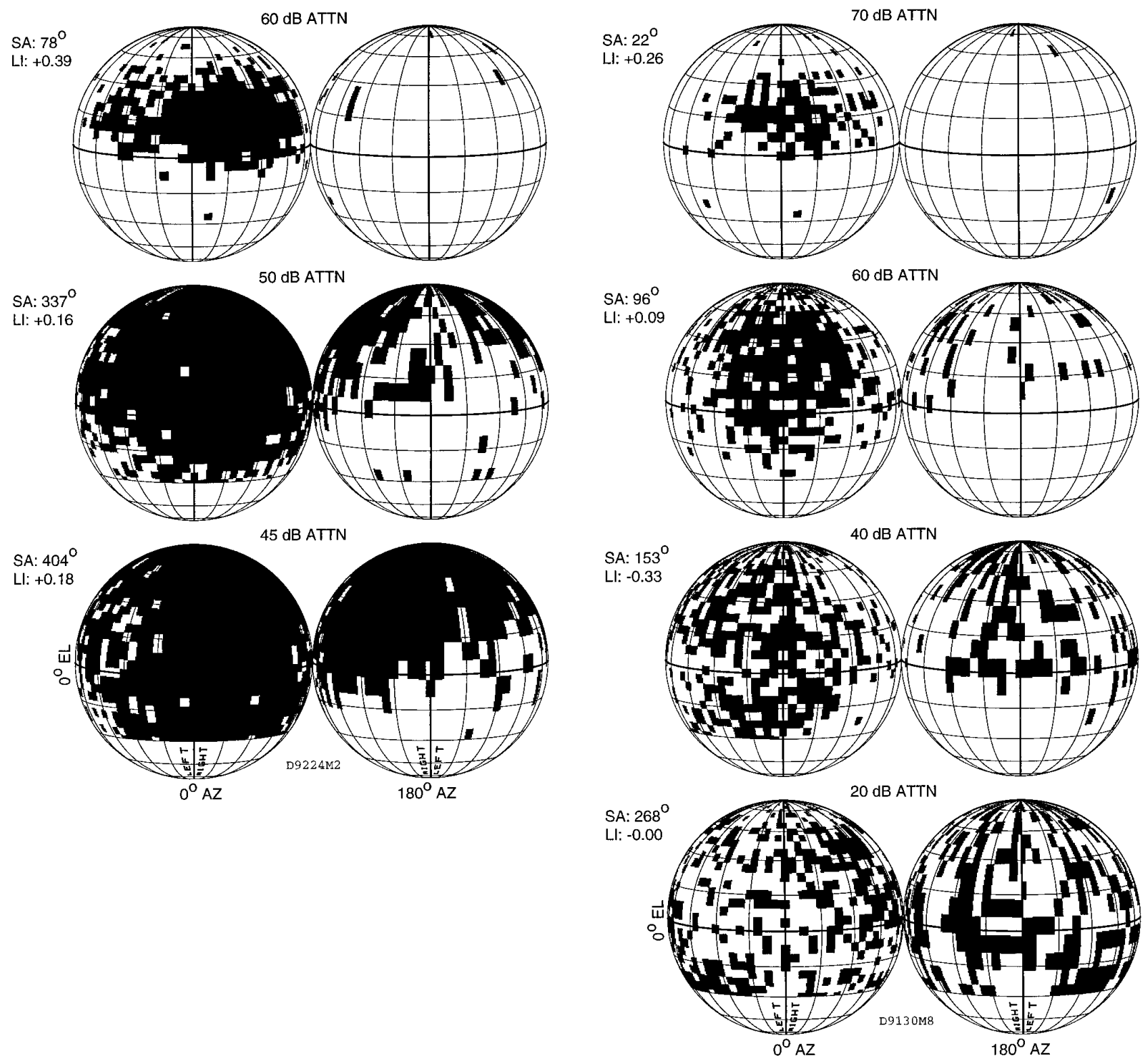

Figure 4. Frontal VSRFs obtained from two AI neurons at different intensities. Neuron on the right exhibits a "fractured" pattern. CF-tone binaural interaction: D9224M2, PB; D9130M8, PB. See text and legend to Figure 1 for further details.

The SMs all tend to cluster around the $18^{\circ}$ line of elevation, with most between $18^{\circ}$ and $72^{\circ}$ of azimuth; the elevational spread is $\sim 15-18^{\circ}$. Except perhaps for the highest and lowest elevations, there is relatively close overlap of the distribution of VSRF centers for this cell population and the acoustic axes; however, when we take into account the frequency dependence of the acoustic axis and the CF of the cell, the relationship is not as clear-cut. In Figure 7, $B$ and $C$, we plot the locus of the SM of each VSRF represented in Figure $7 A$ against that of the acoustic axis at or very near the $C F$ of the cell. The azimuthal $(B)$ and elevational (C) components are plotted separately. The diagonal in each panel indicates a perfect correlation between the center of the VSRF and the direction of the acoustic axis. The experimental data points are distributed rather widely and evenly on either side of the diagonal, both in azimuth and elevation, within the contralateral or ipsilateral upper frontal quadrant of acoustic space.

\section{Internal structure of the VSRF}

The results presented so far have addressed mainly the extent of the spatial domain within which simulated free-field signals influence the output of a cortical neuron. Without exception, spatial tuning of AI neurons in our sample was broad, even at relatively low intensity levels. We thus hypothesized that information about stimulus direction was to be found in the internal structure of the VSRF.

AI neurons responded to our VAS transient signals with but a single spike or short burst of spikes tightly time-locked to the stimulus, and therefore any directional information contained within the VSRF is necessarily to be found in the timing of the discharge or in firing probability or both. Because the full array of our stimulus set consisted of signals from tens of hundreds of closely spaced directions, we were able to derive spatial receptive 


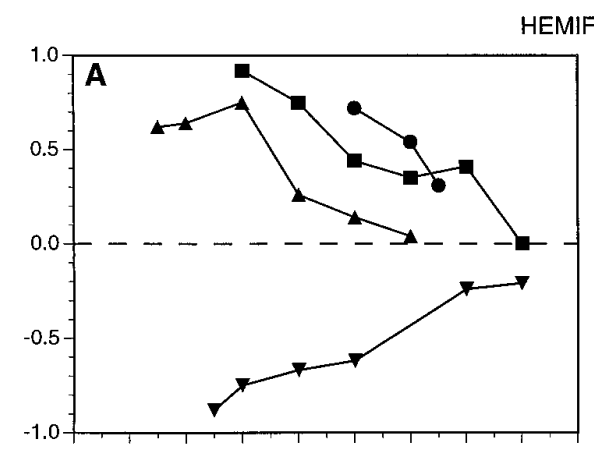

HEMIFIELD - UNBOUNDED

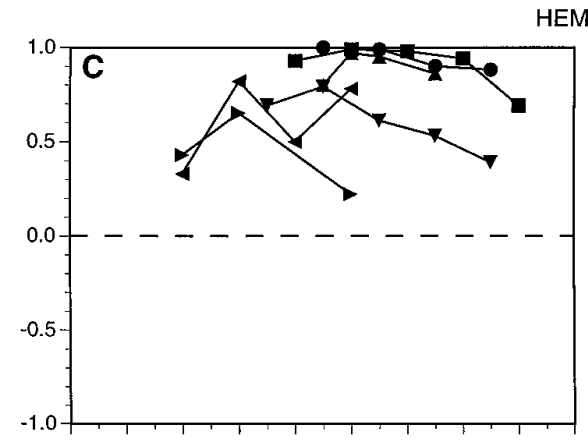

HEMIFIELD - BOUNDED

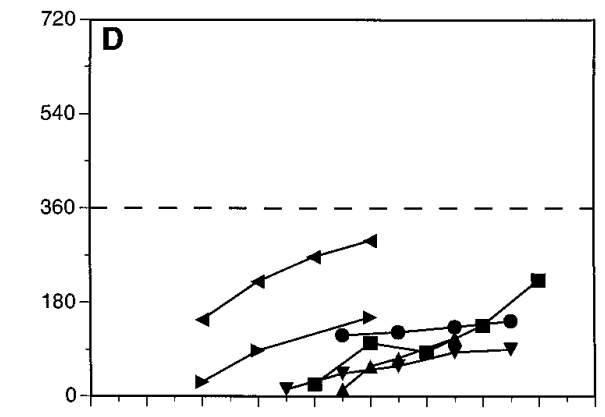

FRONTAL - UNBOUNDED
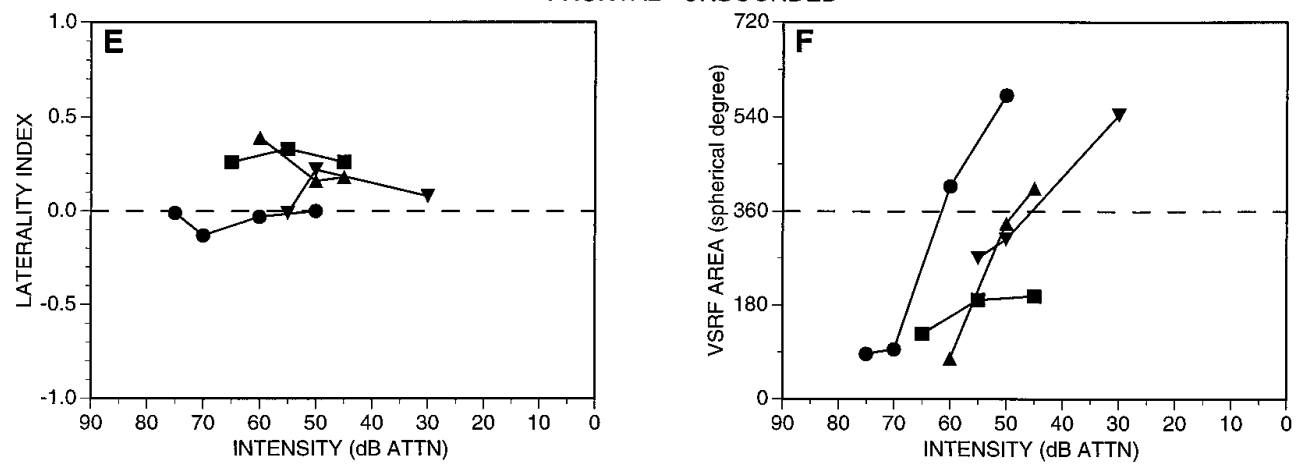

Figure 5. Laterality index and VSRF area plotted as a function of stimulus intensity for 14 neurons. $A, B$, Unbounded VSRFs; $C, D$, bounded VSRFs; $E, F$, frontal VSRFs. See text for further details. fields with a very fine grain and thus to analyze the VSRF for spatial gradients in discharge latency or firing strength or both.

\section{Spike timing: frequency distribution of response latency within the VSRF}

For most VSRFs in our sample, a signal from each VAS direction was presented once. Because AI neurons generated little or no spontaneous activity under the conditions of this experiment, and because we accepted spikes within a narrow (5-50 msec) window of time after stimulus onset, there were few if any spikes in our records that were not associated with a stimulus. At each effective direction, we obtained the latency to the first spike evoked by that stimulus. Figures 8 and 9 illustrate for eleven AI neurons the frequency distribution of first-spike latency within the VSRF, obtained over a range of intensity. The histograms illustrate four observations with respect to spike timing within the VSRF. First, the distribution of response latency across VAS, with few exceptions, was unimodal and sharply peaked (on a time scale measured in milliseconds). Rarely, two peaks appeared (Fig. 8E,F). Second, at any given intensity, response latency typically varied across the VSRF by $\sim 3-5 \mathrm{msec}$; for some cells the spread of latency could be as great as $20 \mathrm{msec}$ (Fig. 9A). Third, increases in intensity most often resulted in systematic shortening of the average response latency (Fig. 8A-F). Usually, average latency was longest near threshold and decreased rapidly at stimulus levels $\sim 20-30 \mathrm{~dB}$ greater than threshold, reaching a near-asymptotic value at the highest intensities used. Latency could shorten, on average, by $\sim 1-5 \mathrm{msec}$ over a range of $10-50 \mathrm{~dB}$. For some neurons illustrated in Figures 8 and 9, an asymptote was not reached. The response latency of a small percentage of cells did not exhibit this same behavior. Examples are shown in Figure 9. Here, increasing intensity resulted in either a lengthening (Fig. $9 A$ ) or little or no systematic change in average latency (Fig. $9 B-E$ ). Fourth, the average latency within the VSRF differed among neurons. Values obtained at highest intensities studied for 87 neurons ranged from 10.2 to $34.3 \mathrm{msec}$.

These findings regarding the frequency distribution of response latency within the VSRF simply revealed that for all neurons studied the spatial receptive fields were not homogeneous with respect to spike timing. Spatial gradients of spike timing were revealed in the spatial distribution of response latency.

Spike timing: spatial distribution of response latency within the VSRF

Figure 10 illustrates for one neuron the highly ordered spatial distribution of response latency commonly seen in our data. The 

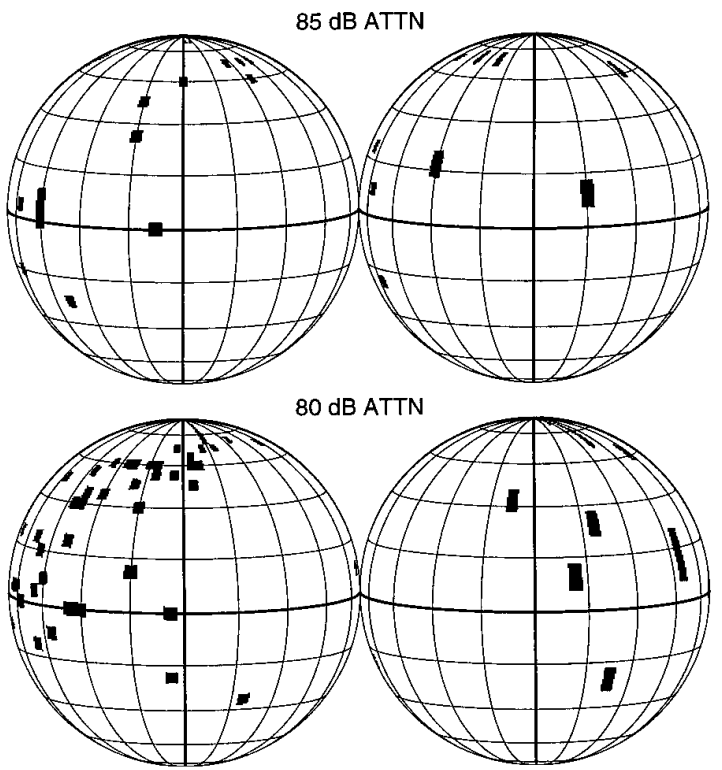

$75 \mathrm{~dB}$ ATTN

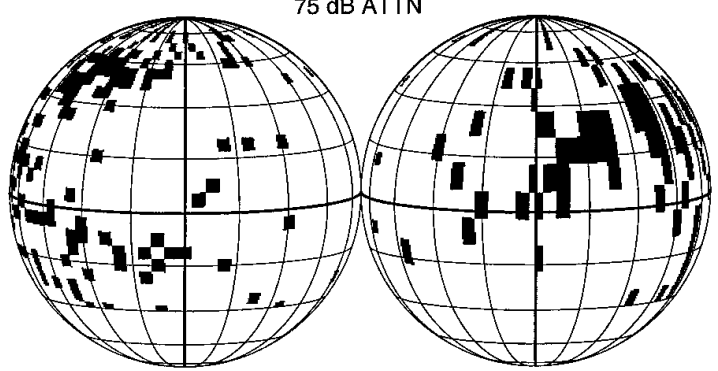

$65 \mathrm{~dB}$ ATTN
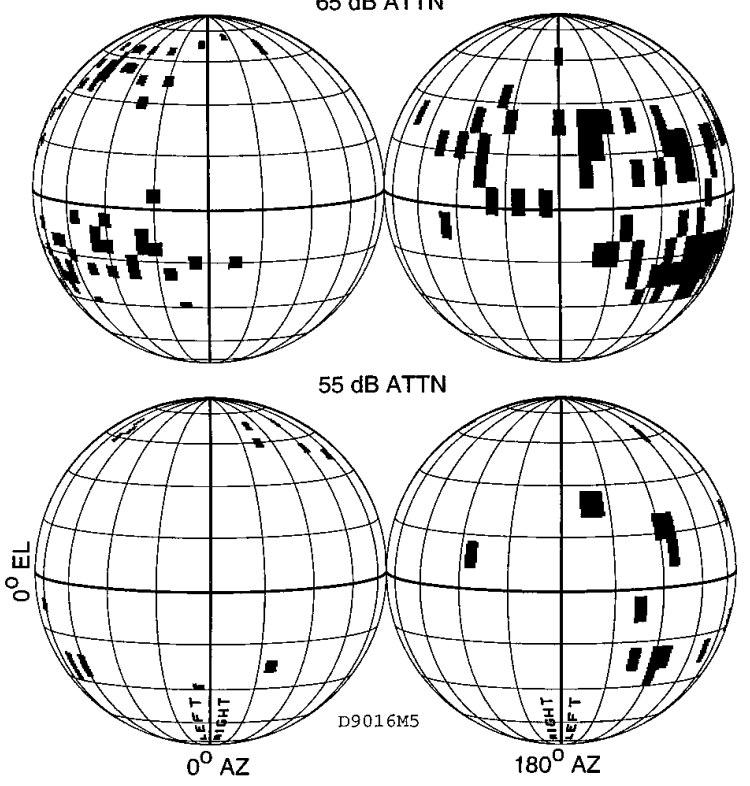

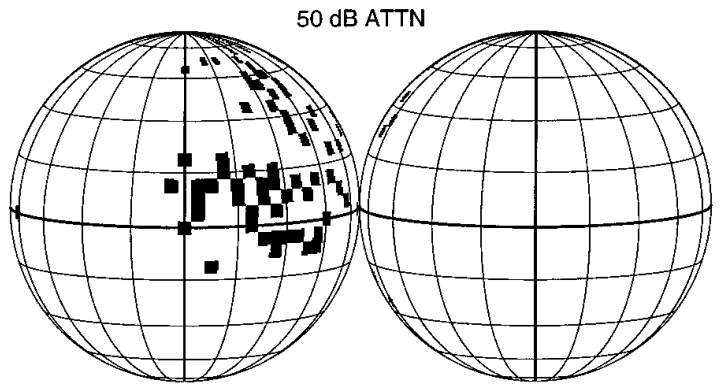

$40 \mathrm{~dB}$ ATTN

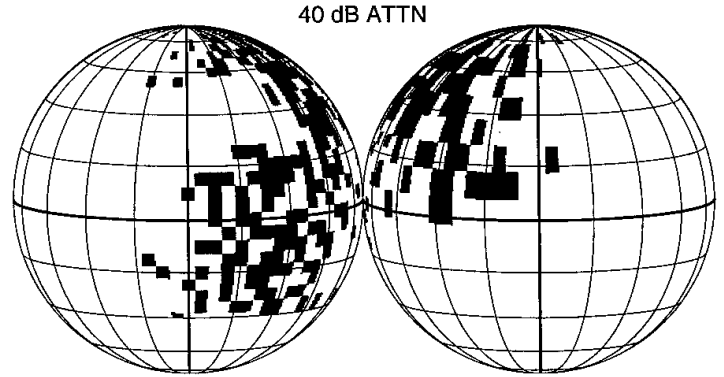

$30 \mathrm{~dB}$ ATTN

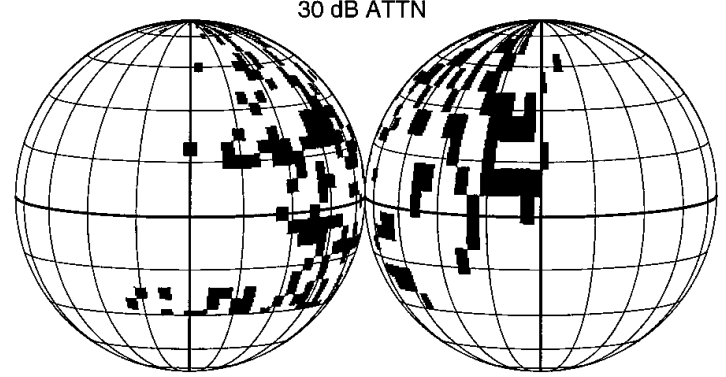

$20 \mathrm{~dB}$ ATTN

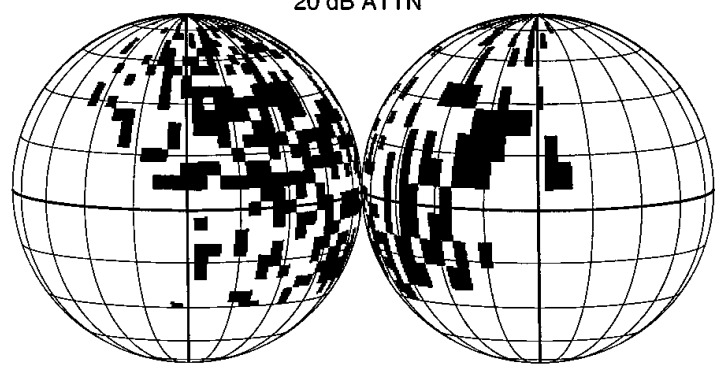

$10 \mathrm{~dB}$ ATTN

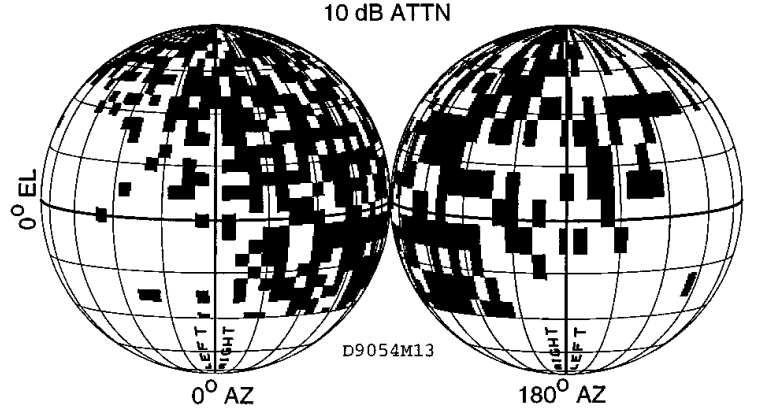

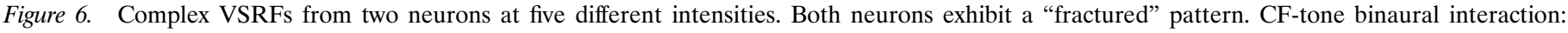
D9016M5, EE; D9054M13, EE. See text and legend to Figure 1 for further details.

results shown were obtained at 5 or $10 \mathrm{~dB}$ intervals over a range of $45 \mathrm{~dB}$ (the spatial tuning properties of this neuron are illustrated in Fig. 1A). The histograms illustrate at each intensity the frequency distribution of first-spike latency, at a resolution of 1 msec; VSRFs illustrate color-coded spatial distributions of latency. Each responsive point in a VSRF has been assigned one of five colors, on the basis of response latency at that point. For VSRFs in the left-hand column, each color corresponds to latencies binned with a fixed $1 \mathrm{msec}$ interval. Thus, all points in the VSRF with a latency between 10.4 and $11.3 \mathrm{msec}$ are red; all points with latency between 11.4 and $12.3 \mathrm{msec}$ are yellow, and so on. The VSRFs in the right-hand column color-code the same 

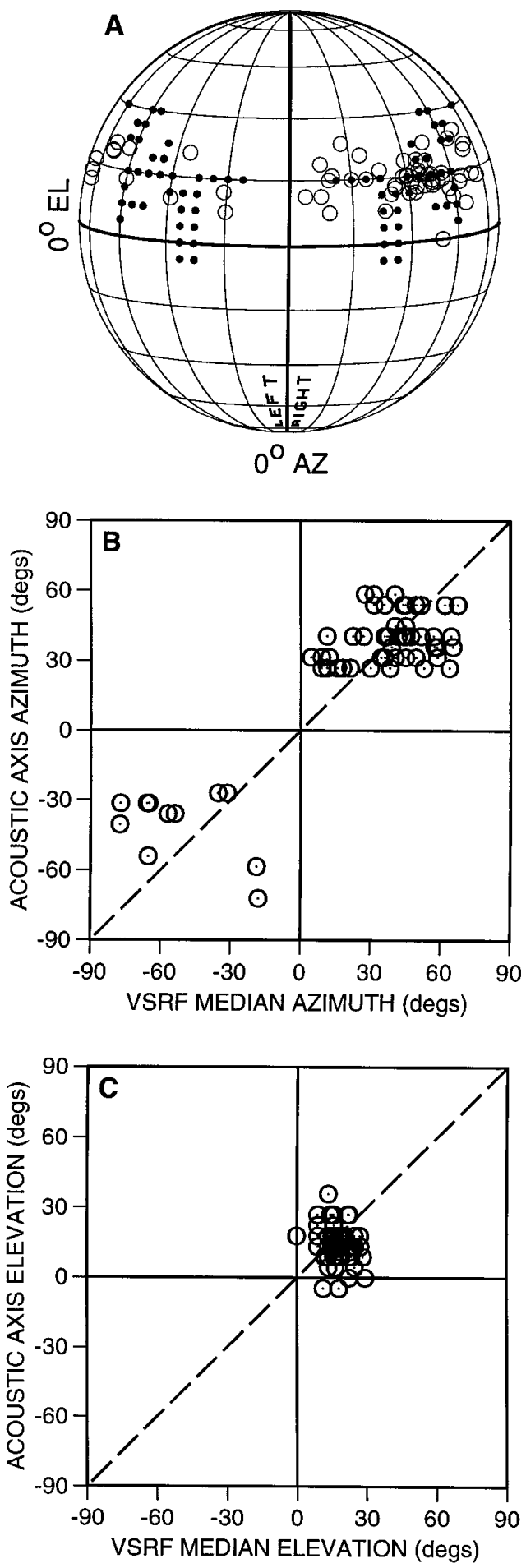

Figure 7. A, Global representation of the distribution of the acoustic axis (filled circles) and the spatial median (open circles) for 65 contralateral and ipsilateral VSRFs. $B$, Scatter plot of acoustic axis versus VSRF median azimuth at CF. Upper right quadrant represents data in the contralateral acoustic hemifield; lower left quadrant represents data in the ipsilateral acoustic hemifield. $C$, Scatter plot of acoustic axis versus VSRF median elevation at CF. Upper right quadrant represents data in the upper right acoustic hemifield.

data using a proportional (quintile) representation of responsive points to determine binwidth. Thus, in the same $5 \mathrm{msec}$ time interval, those points that represent the shortest $20 \%$ of latencies are red; those points representing the next highest $20 \%$ of laten- cies are yellow, and so on. The $5 \mathrm{msec}$ spread of latency values includes all but a small number of effective directions within the VSRF.

Three general observations can be made on the basis of these results. First, there was a systematic intensity-dependent overall expansion of the VSRF, as described earlier. Second, within the VSRF, at each intensity studied there were aggregations of directions that evoked similar response latencies. From these VSRFs it can be seen that the shortest latencies occupy a "core" area in contralateral space on or near the acoustic axis. This core area is surrounded by concentric areas of increasing latency. Third, the absolute value of the latency shortens with increasing intensity, as is shown here by the change in the latency histograms (also see Figs. 8 and 9). In this case, the most common latency mapped into the core near threshold was between 12.4 and 13.4 msec (green), whereas the core was occupied by responses around 10.4-11.4 msec (red) at the highest intensity studied (30 dB ATTN). VSRFs with these properties are referred to as being ordered, and they represent $\sim 40-60 \%$ of our sample.

There was some variability in the spatial distribution of latency among neurons with ordered VSRFs, as illustrated for five cells in Figure 11. VSRFs shown in this figure were obtained near the middle of the dynamic range of the cell and were plotted proportionately, as in Figure 10. The top three VSRFs show a core area of shortest latency in the upper contralateral quadrant of VAS, around the acoustic axis; the fourth VSRF falls in ipsilateral acoustic space. The bottom VSRF in the column is fractured, yet it exhibits a core area confined to the upper frontal quadrant of contralateral acoustic space. Spatial gradients of latency were exhibited by all VSRF classes in our sample population.

From data such as these on VSRFs exhibiting order in spike latency, we are able to conclude that although the overall spatial dimension of the VSRF is large, the core area of shortest latency within the spatial receptive field is far more restricted in size. Just how large this core area is depends on the acceptance criteria used. Our choice of either $1 \mathrm{msec}$ or quintile-based binwidths is rather conservative considering the precise timing of AI spikes in response to our VAS stimuli. Later we will present evidence that the time structure of an ordered VSRF may be correlated with the spatial pattern of discharge probability.

The remaining subset of AI neurons exhibited no such spatial organization to the discharge latency. Instead, latency was distributed rather randomly throughout the VSRF. We refer to such VSRFs as being disordered. Figure 12 illustrates data from one such neuron studied over a range of $45 \mathrm{~dB}$ (its spatial tuning is illustrated in Fig. $1 B$ ). The data are plotted as in Figure 10: absolute latency is plotted in the left-hand column, proportional latency is plotted on the right. The VSRF was relatively small near threshold (65 dB ATTN), occupying an area of virtual space of some 43 spherical degrees on and to the left of the vertical meridian, and the latencies were uniformly long, averaging 14.3 msec. Increasing intensity resulted in a broadening of the VSRF, from 43 spherical degrees to 442 spherical degrees, and a shortening of average latency to $12.1 \mathrm{msec}$. At all intensities studied, the latency ranged over 3-5 msec. Thus, like the ordered VSRF described above, a disordered VSRF was heterogeneous with respect to latency, and the average latency shortened systematically with intensity. Yet, there was little tendency for the spatial loci with similar latency values to aggregate, except perhaps at lowest intensity where all latencies were uniformly long.

Figure 13 illustrates five VSRFs exhibiting temporal disorder, obtained at intensities near the middle of each of the dynamic 

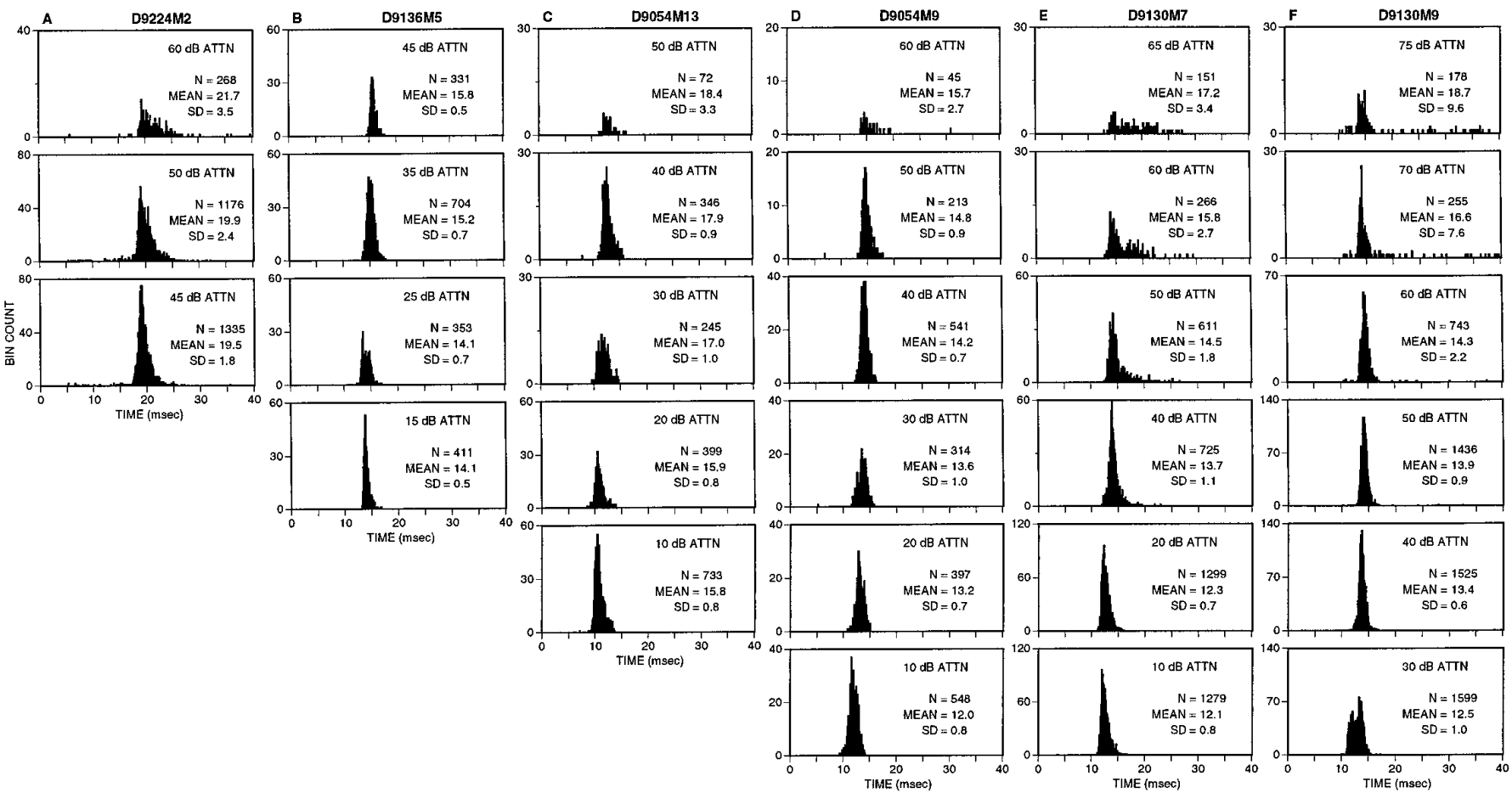

standard deviation $(S D)$ given on each panel.

range of the five cells, showing the variability that existed in our data set with respect to this property. Like ordered VSRFs, disordered fields were exhibited by all classes of VSRFs, whether fractured or nonfractured.

\section{Relationship between response latency and response magnitude}

Data shown so far were derived from experiments in which a single stimulus was presented at each direction in the VAS of the cat. The results of such experiments have provided information on the breadth of spatial tuning, in both azimuth and elevation, and on the internal time structure of the VSRF. They did not reveal, however, how directional information might be contained in the response magnitude of a cell. To evaluate response strength as a possible code for directional hearing, we carried out experiments in which repetitive stimuli were presented at each VAS direction.

In these experiments, we typically used between 5 and 15 stimulus presentations at each VAS direction and used a stimulus set consisting of $\sim 600$ directions. The stimulus set was restricted
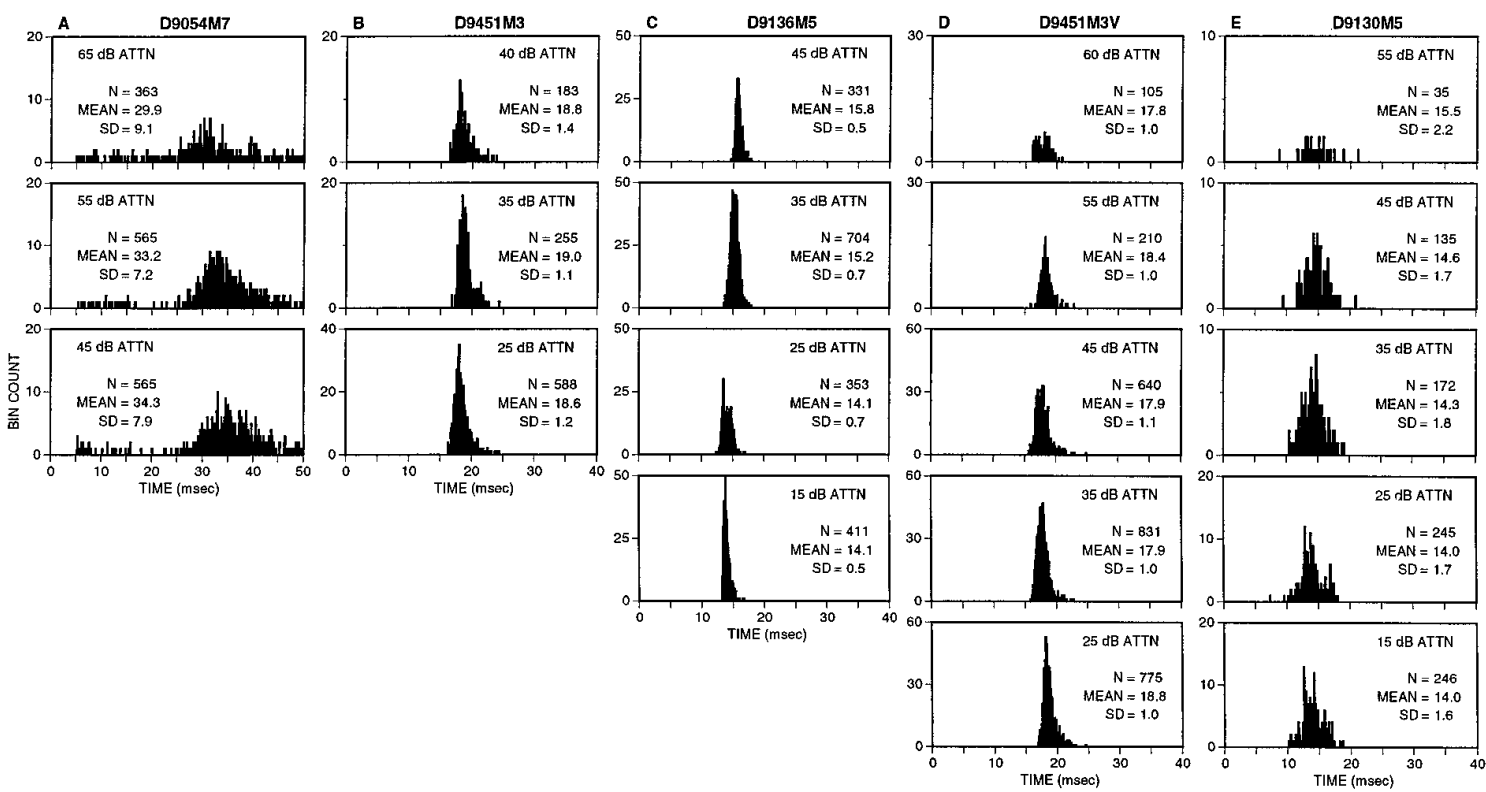

Figure 9. Distribution of first-spike latency for five AI neurons at different intensities. See legend to Figure 8 for further details. 


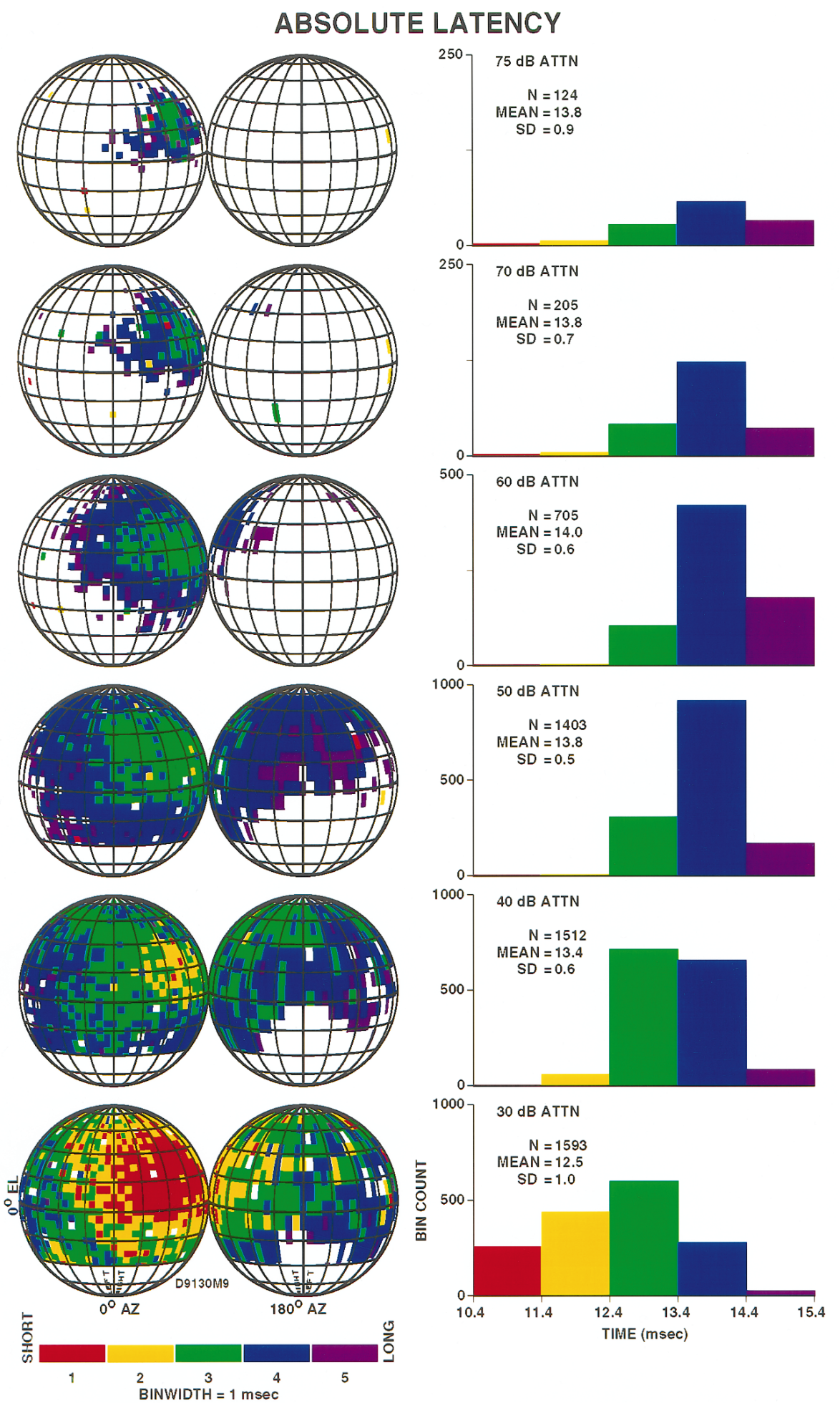

\section{PROPORTIONAL LATENCY}
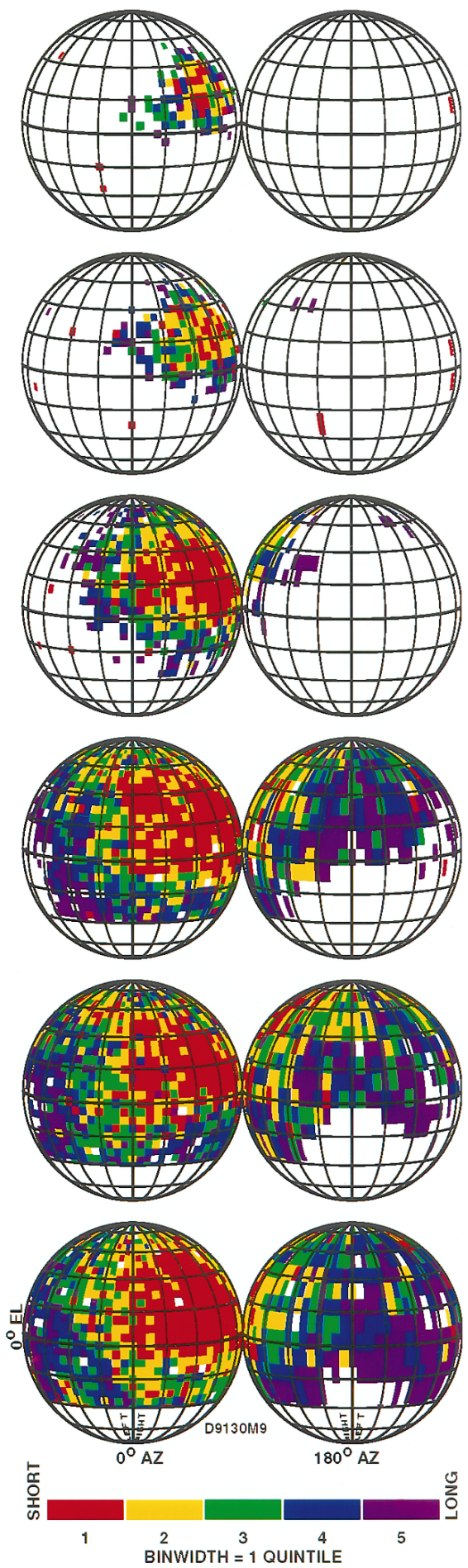

Figure 10. "Ordered" VSRFs based on first-spike latency from one AI neuron obtained at six different intensities. Histograms show the frequency distribution of latency at each intensity. Left column, VSRFs color-coded for an absolute latency within a fixed binwidth of 1 msec. Colors in the VSRFs correspond to colors of bins in accompanying color bar and latency histogram. Right column, VSRFs based on the same data but plotted such that binwidth represents an equal (quintile) proportion of latency values. Colors in the VSRFs correspond to the colors of the accompanying color bar. CF-tone binaural interaction: D9130M9, EI. See text for further details.

in size because the data collection time using multiple trials at each direction was extended by several hours beyond that necessary to obtain single-trial VSRFs. For each neuron studied, we first obtained one or more single-repetition VSRFs, which in turn guided the choice of the size of the stimulus set used for subsequent multiple-trial mapping. The multiple-trial stimulus set was typically presented at one intensity, near the center of the dynamic range of the cell. This approach provided data sufficient to esti- 

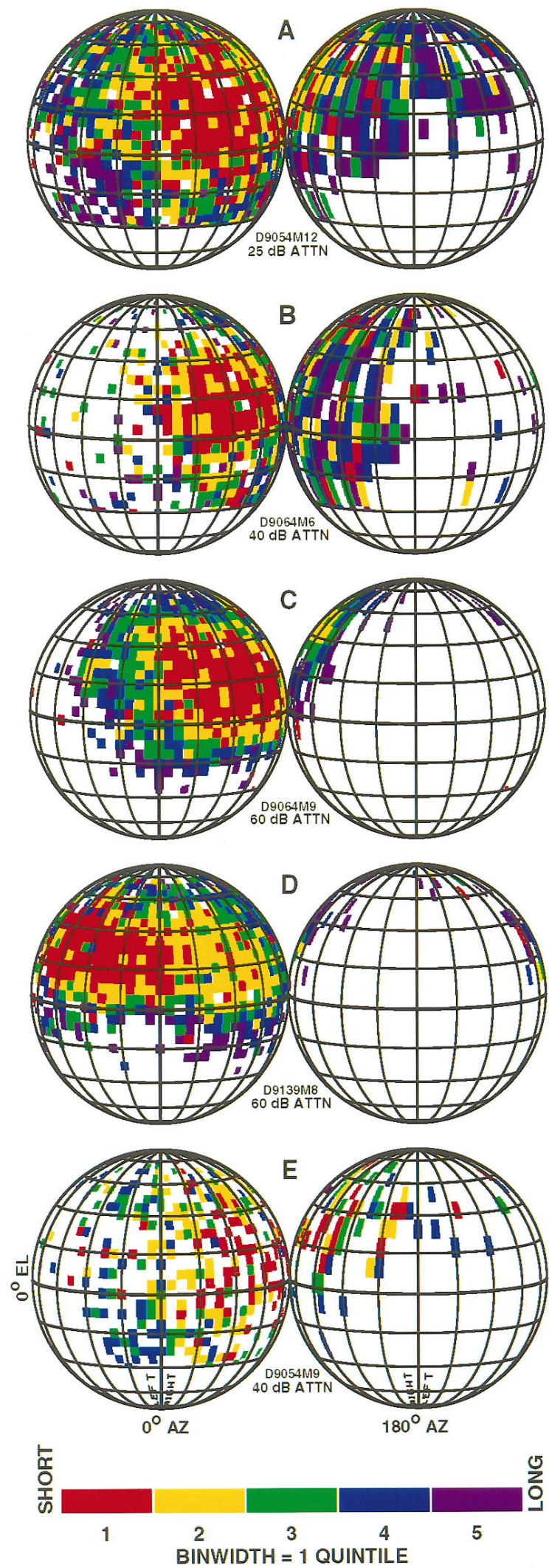

Figure 11. "Ordered" VSRFs based on first-spike latency from five AI neurons, illustrating the range of VSRFs within that category. Intensity shown below each panel. CF-tone binaural interactions: D9054M12, EE; D9064M6, EI; D9064M9, EE; D9139M8, EE; D9054M9, EE. See legend to Figure 10 for additional details. mate the spatial distribution of response magnitude and of the possible relationship between response magnitude and response latency within the VSRF. Because these cells respond with a single spike at many effective directions, firing probability and spike count are often equivalent measures of response strength in the receptive field.

The VSRFs illustrated in Figure 14 were derived from the responses of five neurons to 15 stimuli at each of the tested directions. The data are plotted proportionately (quintiles), as in Figure 10; the distribution of mean first-spike latency is shown on the right, and the distribution of firing probability is shown on the left. Firing probability is based on the number of effective trials from 15 stimulus presentations at each direction. Between each pair of VSRFs is a scatter plot of mean first-spike latency versus firing probability, with the median of the mean latency distribution at each firing probability shown as an open circle. The Spearman test for rank correlation was used to test the association between the ordinal measure of firing probability and the continuous dependent variable of mean latency. The median of the mean latency values at each firing probability was used in the test statistic to better approximate the assumption that the sample of pairs was random.

The VSRF illustrated in Figure $14 A$ is disordered in latency and in firing probability, as evidenced by the near-random distribution of colors in both VSRF maps. The rank correlation coefficient was not significant (0.05 level; two-tail test). VSRFs from three neurons that exhibited an ordered spatial organization of response magnitude and latency are illustrated in Figure $14 B-D$. In such cells, a core of the VSRF can be identified containing those directions that evoked the highest firing probability or shortest response latencies. The maps took different forms in different cells (also see Fig. 11), but for a given neuron a profile defined by response strength or latency seemed similar by simple inspection of the VSRFs. For example, the cores for the neuron in Figure $14 B$ seem to consist of a single focus in the contralateral frontal quadrant, regardless of whether response strength or latency is mapped. Additionally, there is a clear overlap of the VSRF areas containing the cores. By comparison, the core of the VSRFs for the cell shown in Figure $14 D$ seems to consist of two foci. The laterally positioned focus is more elongated in elevation than its medially located counterpart. Scatterplots suggest an association between response strength and latency within ordered VSRFs; the shortest latencies tended to occur at directions where the firing probability is highest, whereas the longest latencies encountered within the receptive field tended to occur at directions evoking the weakest responses. The rank correlation coefficient was significant (0.01 level; two-tail test) for all three cells. Finally, Figure $14 E$ illustrates VSRFs that at the intensity studied showed an ordered firing probability but no sign of order in latency. The rank correlation coefficient was not significant ( 0.05 level; two-tail test). This neuron, when studied with tonal stimuli, also showed a strong nonmonotonic relationship between the response magnitude and SPL.

\section{DISCUSSION}

Neurons in AI exhibit spatial receptive field properties that could aid in signaling the direction, or change in direction, of a brief broad-band sound. Near threshold, the passive acoustical properties of the head and pinnae place the receptive field on or near the acoustic axis. At moderate to high intensities, binaural interactions affect the size and location of the receptive field. Within a 


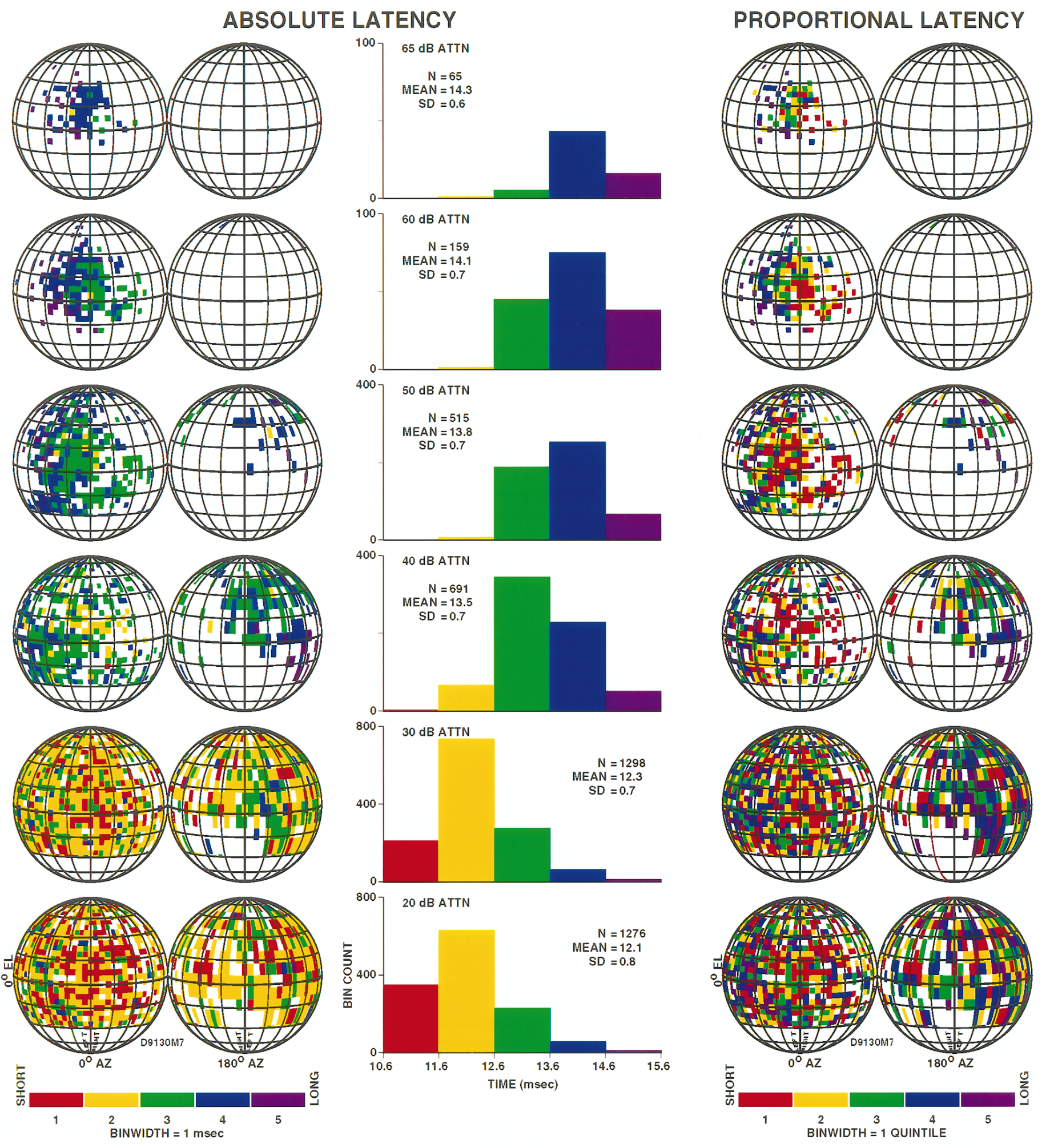

Figure 12. "Disordered" VSRFs based on first-spike latency from one AI neuron obtained at six different intensities. CF-tone binaural interaction: D9130M7, EE. See legend to Figure 10 and text for further details.

receptive field, there may exist a spatial gradient of response time or response magnitude or both.

We observed a strong head and pinna effect. At $10-30 \mathrm{~dB}$ above threshold, the distribution of centers of VSRFs was nearly coextensive with the distribution of acoustic axes over the CF range of our cell population. When CF was taken into account, the corre- spondence was found to be weaker, which agrees with earlier results from AI (Middlebrooks and Pettigrew, 1981) and ICC (Semple et al., 1983). This may be accounted for by the fact that in a VAS the spatial distribution of high-frequency stimulus amplitude is rarely symmetric about its maximum amplitude (i.e., acoustic axis). Thus, at low intensities, high-CF neurons probably 

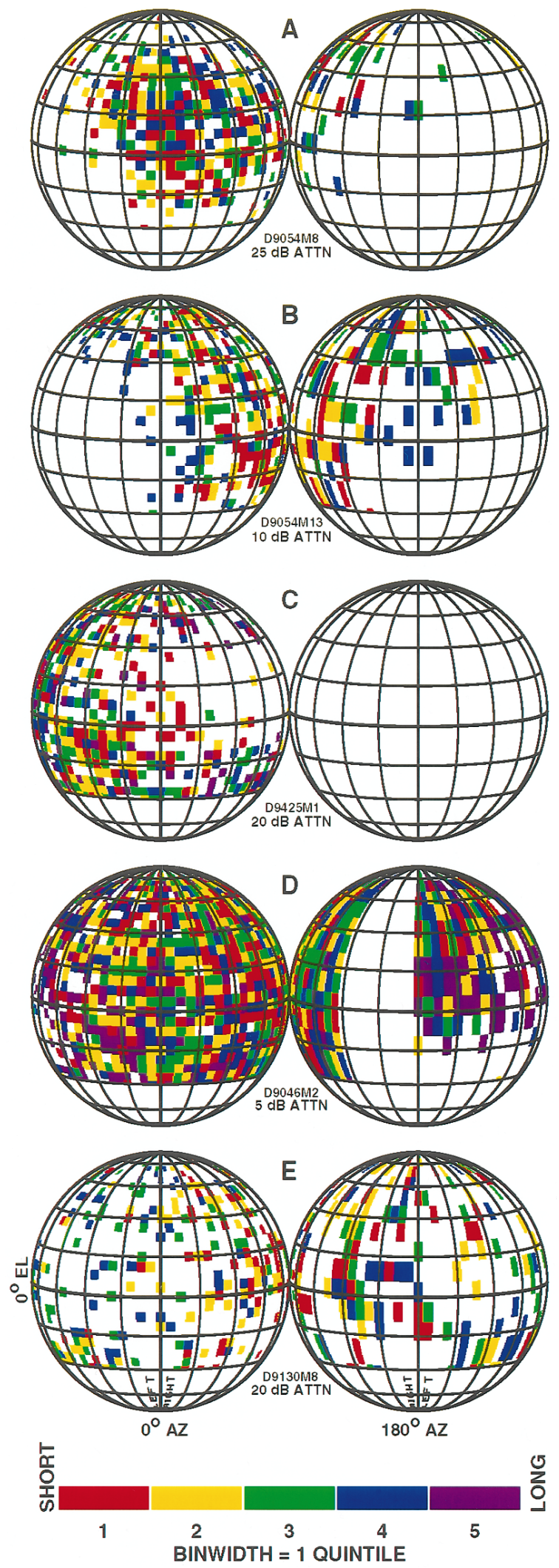

Figure 13. "Disordered" VSRFs based on first-spike latency from five AI neurons, illustrating the range of VSRFs within that category. Intensity shown below each panel. CF-tone binaural interactions: D9054M8, EE; D9054M13, EE; D9425M1, PB; D9064M2, EI; D9130M8, PB. See legend to Figure 11 for additional details. integrate stimulus energy from those asymmetrically distributed directions (Brugge et al., 1994). At higher intensities, the acoustic axis for a subset of AI neurons was associated with a "core" of the spatial receptive field representing shortest latency and in some cases greatest response magnitude. Thus, for sounds allowing auditory feedback during head or pinnae movements, information about changes in intensity and spectrum would be available to bring into coarse alignment the acoustic axis of one ear and the direction of the sound. The signal-to-noise ratio would also improve, thereby possibly improving signal detectability (Middlebrooks and Pettigrew, 1981; Semple et al., 1983). For a single brief sound providing no opportunity for auditory feedback, the VSRF cues described would have limited value to the animal in orienting to or localizing a sound in space. Under these conditions other mechanisms must be engaged, for cats seem quite capable of orienting in the appropriate direction when a single brief noise is introduced into the sound field (Beitel and Kaas, 1993; Populin and Yin, 1995).

Spatial receptive fields of most recorded AI neurons expanded their borders monotonically with increases in stimulus intensity. Middlebrooks and Pettigrew (1981) showed that spatial tuning of cat AI neurons expanded to the midline, or slightly beyond, when stimulus intensity was raised by as much as $30 \mathrm{~dB}$ above threshold. Imig et al. (1990) and Rajan et al. (1990) reported that azimuthal functions widened with increasing intensity, but this was often limited to a frontal acoustic quadrant by binaural interactions (Samson et al., 1994). We demonstrated previously that input to neurons exhibiting "unbounded" VSRFs was dominated by excitatory processes; for "bounded" VSRFs, a strong net inhibitory input was restricted largely to one or the other acoustic hemifield (Brugge et al., 1994, 1996). Thus, from the standpoint of neurons with "bounded" receptive fields, a brief sound is lateralized to one or the other acoustic hemifield over a wide range of intensity. Other neurons with VSRFs confined to the frontal midline would be capable of detecting a sound arriving from ahead of the animal, at least at moderate intensity where binaural interactions are at play and the VSRFs are still restricted (see Samson et al., 1994). Such mechanisms may be used for orienting to a single brief sound or to trains of acoustic transients.

The finding that discharge properties vary across a spatial receptive field has been made by others in auditory cortex (Eisenman, 1974; Sovijärvi and Hyvärinen, 1974; Knudsen et al., 1977; Middlebrooks et al., 1994) and ICC (Bock and Webster, 1974; Moore et al., 1984a,b). The most detailed studies show that the magnitude of spike discharge changes systematically along the azimuth for the majority of recorded AI neurons (Imig et al., 1990; Rajan et al., 1990a,b). Within the anterior ectosylvian auditory field, the temporal pattern of discharge changes with azimuthal location and carries sufficient information to encode sound-source direction (Middlebrooks et al., 1994).

Using our VAS paradigm to probe the internal structure of the entire receptive field with high spatial resolution, we found that for a sizable population of recorded AI neurons discharge timing was distributed in an orderly way within the VSRF. Shortest latencies tended to occupy a core of the VSRF, which fell on or near the acoustic axis. At greater eccentricity, the latency lengthened progressively, and a spatial gradient representing time was established. For some cells, a similar gradient was established for response magnitude. We referred previously to the core as the "effective receptive field" (Brugge et al., 1996), which may correspond to what Knudsen and Konishi (1978) called the "best area" of spatial receptive fields of neurons in the midbrain of the barn 

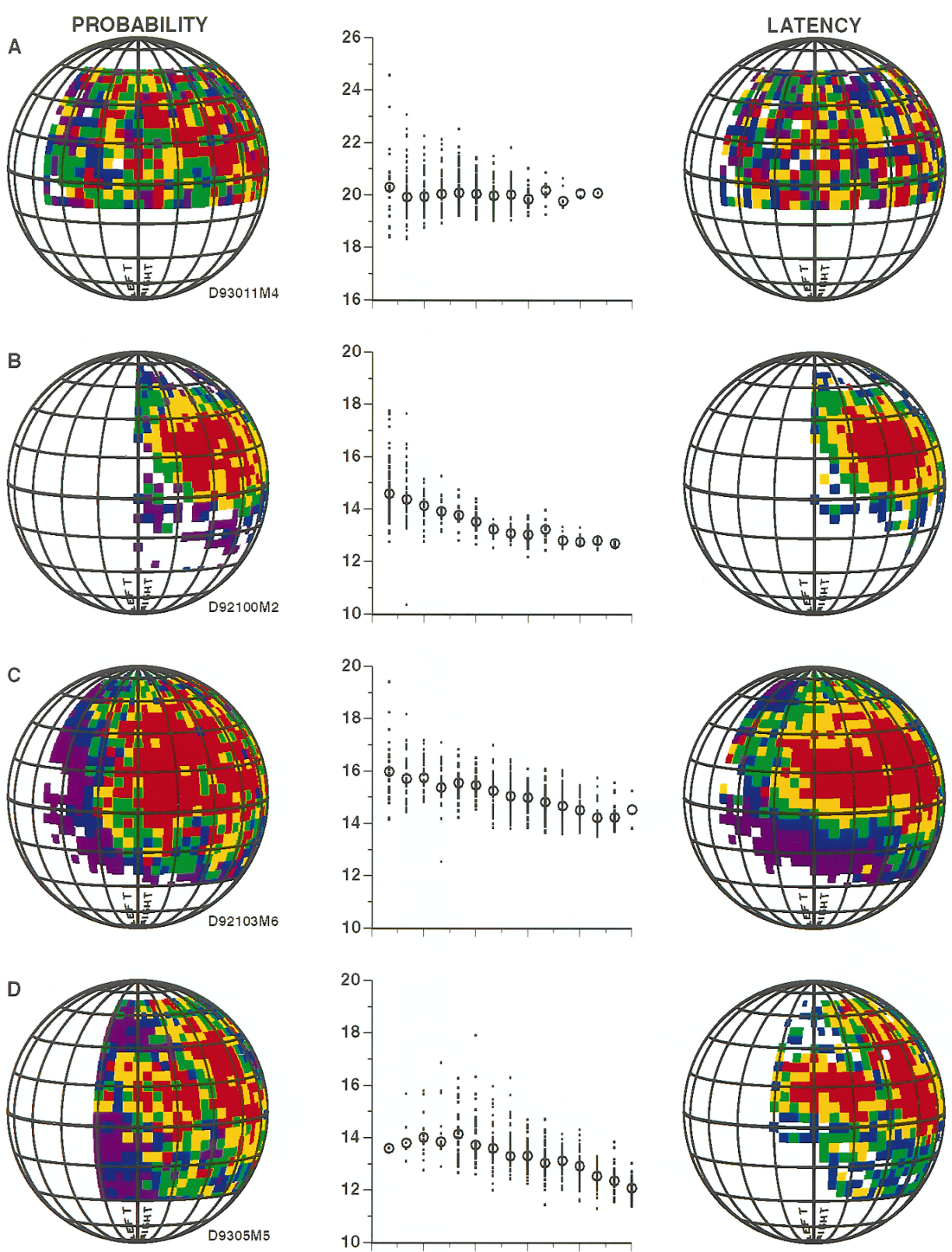

Figure 14. VSRFs based on proportional latency (right column) and proportional firing probability (left column) in response to 15 stimulus trials at each VAS direction. Scatter plots show relationship between mean first spike latency and firing probability at each effective point in the corresponding VSRFs. Only a portion of the standard 1650 loci were tested in each case $(A, n=525 ; B, n=654 ; C, n=819$; $D, n=575 ; E, n=554)$. CF-tone binaural interactions: D93011M4, PB; D92100M2, unknown; D92103M6, EE; D9305M5, EE; D92100M4, EE. See text for further details.
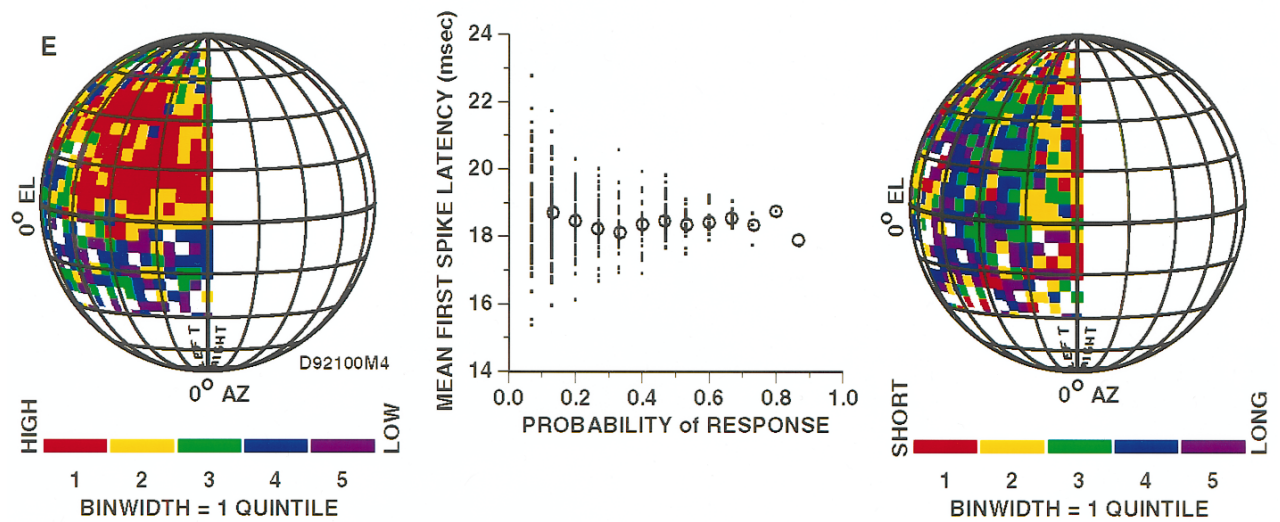

owl. The size of this core may be highly focused, depending on the acceptance criteria used (Brugge et al., 1996).

The average latency varied by as much as $24 \mathrm{msec}$ across our neuron population. For the great majority of cells, as stimulus intensity was raised over a range of some $40-50 \mathrm{~dB}$, the response latency shortened progressively by as much as $1-5 \mathrm{msec}$ at each effective VSRF direction. The core area remained relatively constant in size and location, however, and the gradient of latency was 
preserved. This may be related to a listener's constancy in directional hearing over a range of stimulus intensity (Yost and Hafter, 1987). Because latency shifts with intensity, it is likely that relative rather than absolute latency could serve as one coding mechanism involved in detecting the direction, or change in direction, of a transient sound. A related code may be relative response magnitude, because firing probability or spike count may correlate with latency.

Using the same VAS paradigm, it has been shown that the spatial distribution of stimulus amplitude is transmitted to the brain in the discharge patterns across the auditory nerve array (Poon and Brugge, 1993). This spatial response pattern may be retained by certain neurons of AI, because a similar relationship seems to exist between the location and shape of their VSRF and the passive acoustical properties of the head and pinna (Reale et al., 1991; Brugge et al., 1994). Thus, functional gradients such as these in AI receptive fields might be expected when there is a potent monaural input to the cell, considering the systematic changes in onset latency and spike count that accompany changes in sound intensity (Phillips and Hall, 1990; Phillips, 1993a,b). The fact that some AI neurons show no evidence of functional gradients in the their VSRFs, however, indicates that the internal structure of an AI spatial receptive field need not be determined only by this spatial distribution of stimulus intensity. Other factors, including interaural time, intensity, and spectrum may be playing major roles in determining receptive field properties. We have not yet studied the effects of varying interaural parameters, and our multiple-trial data are not adequate to carry out the detailed analysis of spatial patterns necessary to settle the issue of how closely receptive field structure is related to these spatial cues. Although neurons with disordered VSRFs may code direction by the location of their VSRFs near threshold and in their binaural interactions, there is seemingly no additional directional information to be found in the internal structure of their receptive fields.

Large receptive fields have long been encountered in the visual cortex (for review, see McIlwain, 1976; Maunsell and Newsome, 1987; Dinse et al., 1991). Their internal structure, like that of AI neurons, may be based on response strength and timing (Bear et al., 1971; Sasaki et al., 1971a,b; Henry, 1977; Palmer and Davis, 1981; Reinis et al., 1988), resulting in gradients that may account for many of the static and dynamic response properties exhibited by visual cortical neurons (Palmer and Davis, 1981; Albrecht and Geisler, 1991; Reid et al., 1991; Tolhurst and Dean, 1991; Jagadeesh et al., 1993). Simple cells in the primary visual cortex of the cat exhibit a latency gradient across the receptive field such that fluctuations in membrane potential evoked by moving stimuli are predicted accurately by the linear summation of the temporal response properties to stationary stimuli (Jagadeesh et al., 1993). Reinis et al. (1988) reported that visual neurons of cat cortical area 18 fire repeatedly only when the visual stimulus is present at precisely defined locations within the receptive field, and they postulated that a population of such neurons could detect a moving image. If gradients in latency and response strength across AI receptive fields are operating similarly, then we would predict that such a neuron would exhibit sensitivity to a train of transient sounds that produce so-called "apparent sound motion" (Perrott, 1974, 1982). When one considers that the cat is able to make independent movements of the two pinnae, it is possible that a high degree of localization ability could be achieved by such temporalsummation mechanisms operating in ensembles of AI cells. We are currently using the VAS approach in studies of dynamic mechanisms of spatial hearing.

\section{REFERENCES}

Albrecht DG, Geisler WS (1991) Motion selectivity and the contrastresponse function of simple cells in the visual cortex. Vis Neurosci 7:531-546.

Bear DM, Sasaki H, Frank RE (1971) Sequential changes in receptive fields of striate neurons in dark adapted cats. Exp Brain Res $13: 256-272$

Beitel RE, Kaas JH (1993) Effects of bilateral and unilateral ablation of auditory cortex in cats on the unconditioned head orienting response to acoustic stimuli. J Neurophysiol 70:351-369.

Benson DA, Hienz RD, Goldstein Jr MH (1981) Single-unit activity in the auditory cortex of monkeys actively localizing sound sources: spatial tuning and behavioral dependency. Brain Res 219:249-267.

Bock GR, Webster WR (1974) Coding of spatial location by single units in the inferior colliculus of the alert cat. Exp Brain Res 21:387-398.

Brugge JF, Reale RA (1985) Auditory cortex. In: Cerebral cortex, Vol 4, Association and auditory cortices (Peters A, Jones EG, eds), pp 229271. New York: Plenum.

Brugge JF, Reale RA, Hind JE (1996) Auditory cortex and spatial hearing. In: Conference on binaural and spatial hearing (Gilke R, Anderson T eds). Hillsdale, NJ: Earlbaum.

Brugge JF, Dubrovsky NA, Aitkin LM, Anderson DJ (1969) Sensitivity of single neurons in auditory cortex of cat to binaural tonal stimulation: effects of varying interaural time and intensity. J Neurophysiol 32:1005-1024.

Brugge JF, Reale RA, Hind JE, Chan JC, Musicant AD, Poon PW (1994) Simulation of free-field sound sources and its application to studies of cortical mechanisms of sound localization in the cat. Hear Res 73:67-84.

Chan JCK, Musicant AD, Hind JE (1993) An insert earphone system for delivery of spectrally shaped signals for physiological studies. J Acoust Soc Am 93:1496-1501.

Chen J, Van Veen BD, Hecox KE (1995) A spatial feature extraction and regularization model for the head-related transfer function. $\mathrm{J}$ Acoust Soc Am 1:439-452.

Chen J, Wu Z, Reale RA (1994) Applications of least-squares FIR filters to virtual acoustic space. Hear Res 80:153-166.

Clarey JC, Barone P, Imig TJ (1992) Physiology of thalamus and cortex. In: The mammalian auditory pathway: neurophysiology (Popper AN, Fay RR, eds), pp 232-334. New York: Springer.

Dinse HR, Krüger K, Mallot HA, Best J (1991) Temporal structure of cortical information processing: cortical architecture, oscillations, and non-separability of spatio-temporal receptive field organization. In: Neuronal cooperativity (Krüger J, ed), pp 68-104. Berlin: Springer.

Eisenman LM (1974) Neural encoding of sound location: an electrophysiological study in auditory cortex (AI) of the cat using free field stimuli. Brain Res 75:203-214.

Fisher NI, Lewis T, Embleton BJJ (1987) Statistical analysis of spherical data. Cambridge: Cambridge UP.

Henry GH (1977) Receptive field classes in the striate cortex of the cat. Brain Res 133:1-28.

Imig TJ, Irons WA, Samson FR (1990) Single-unit selectivity to azimuthal direction and sound pressure level of noise bursts in cat highfrequency primary auditory cortex. J Neurophysiol 63:1448-1466.

Jagadeesh B, Wheat HS, Ferster D (1993) Linearity of summation of synaptic potentials underlying direction selectivity in simple cells of the cat visual cortex. Science 262:1901-1904.

Knudsen EI, Konishi M (1978) A neural map of auditory space in the owl. Science 200:795-797.

Knudsen EI, Konishi M, Pettigrew JD (1977) Receptive fields of auditory neurons in the owl. Science 198:1278-1280.

Maunsell JHR, Newsome WT (1987) Visual processing in monkey extrastriate cortex. Annu Rev Neurosci 10:363-401.

McIlwain JT (1976) Large receptive fields and spatial transformations in the visual system. In: International review of physiology (Porter R, ed), pp 242-247. Baltimore: University Park.

Merzenich MM, Knight PL, Roth GL (1975) Representation of cochlea within primary auditory cortex in the cat. J Neurophysiol 38:231-249.

Middlebrooks JC, Pettigrew JD (1981) Functional classes of neurons in primary auditory cortex of the cat distinguished by sensitivity to sound location. J Neurosci 1:107-120. 
Middlebrooks JC, Clock AE, Xu L, Green DM (1994) A panoramic code for sound location by cortical neurons. Science 264:842-844.

Moore DR, Hutchings ME, Addison PD, Semple MN, Aitkin LM (1984b) Properties of spatial receptive fields in the central nucleus of the cat inferior colliculus. II. Stimulus intensity effects. Hear Res 13:175-188.

Moore DR, Semple MN, Addison PD, Aitkin LM (1984a) Properties of spatial receptive fields in the central nucleus of the cat inferior colliculus. I. Responses to tones of low intensity. Hear Res 13:159-174.

Musicant AD, Chan JCK, Hind JE (1990) Direction-dependent spectral properties of cat external ear: new data and cross-species comparisons. J Acoust Soc Am 87:757-781.

Palmer LA, Davis TL (1981) Receptive field structure in cat striate cortex. J Neurophysiol 46:260-276.

Perrott DR (1974) Auditory apparent motion. J Audit Res 3:163-169.

Perrott DR (1982) Studies in the perception of auditory motion. In: Localization of sound: theory and applications (Gatehouse RW ed), pp 169-193. Groton, CT: Amphora.

Phillips DP (1993a) Representation of acoustic events in the primary auditory cortex. J Exp Psychol Hum Percept Perform 19:203-216.

Phillips DP (1993b) Neural representation of stimulus times in the primary auditory cortex. Ann NY Acad Sci 682:104-118.

Phillips DP (1995) Central auditory processing: a view from auditory neuroscience. Am J Otol 16:338-352.

Phillips DP, Hall SE (1990) Response timing constraints on the cortical representation of sound time structure. J Acoust Soc Am 88:1403-1411.

Phillips DP, Irvine DR (1981) Responses of single neurons in physiologically defined area AI of cat cerebral cortex: sensitivity to interaural intensity differences. Hear Res 4:299-307.

Poon PWF, Brugge JF (1993) Virtual space receptive fields of single auditory nerve fibers. J Neurophysiol 70:667-676.

Populin LC, Yin TCT (1995) Behavioral studies of sound localization in the cat. Soc Neurosci Abstr 21:129.

Rajan R, Aitkin LM, Irvine DRF, McKay J (1990a) Azimuthal sensitivity of neurons in primary auditory cortex of cats. I. Types of sensitivity and the effects of variations in stimulus parameters. $J$ Neurophysiol 64:872-887.

Rajan R, Aitkin LM, Irvine DRF (1990b) Azimuthal sensitivity of neurons in primary auditory cortex of cats. II. Organization along frequency-band strips. J Neurophysiol 64:888-902.

Reale RA, Brugge JF (1990) Auditory cortical neurons are sensitive to static and continuously changing interaural phase cues. J Neurophysiol 64:1247-1260.

Reale RA, Imig TJ (1980) Tonotopic organization in auditory cortex of the cat. J Comp Neurol 192:265-291.
Reale RA, Brugge JF, Chan JCK, Musicant AD, Hind JE, Poon PWF (1991) Monaural direction-dependent responses of neurons in cat primary auditory (AI) cortex. Soc Neurosci Abstr 17:449.

Reale RA, Chen J, Hind JE, Brugge JF (1996) An implementation of virtual acoustic space for neurophysiological studies of directional hearing. In: Virtual auditory space: generation and application (Carlile S, ed), pp 153-183. Georgetown, TX: Landes.

Reid RC, Soodak RE, Shapley RM (1991) Directional selectivity and spatiotemporal structure of receptive fields of simple cells in cat striate cortex. J Neurophysiol 66:505-529.

Reinis S, Weiss DS, Landolt JP (1988) Lack of homogeneity of receptive fields of visual neurons in the cortical area 18 of the cat. Biol Cyber 59:41-48.

Rice JJ, May BJ, Spirou GA, Young ED (1992) Pinna-based spectral cues for sound localization in cat. Hear Res 58:132-152.

Samson FK, Barone P, Clarey JC, Imig TJ (1994) Effects of ear plugging on single-unit azimuth sensitivity in cat primary auditory cortex. II. Azimuth tuning dependent upon binaural stimulation. J Neurophysiol 71:2194-2216.

Sasaki H, Bear DM, Ervin FR (1971a) Quantitative characterization of unit response in the visual system. Exp Brain Res 13:239-255.

Sasaki H, Saito Y, Bear DM, Ervin FR (1971b) Quantitative variation in striate receptive fields of cats as a function of light and dark adaptation. Exp Brain Res 13:273-293.

Semple MN, Aitkin LM, Calford MB, Pettigrew JD, Phillips DP (1983) Spatial receptive fields in the cat inferior colliculus. Hear Res 10:203-215.

Sovijärvi ARA, Hyvärinen J (1974) Auditory cortical neurons in the cat sensitive to the direction of sound source movement. Brain Res 73:455-471.

Tolhurst DJ, Dean AF (1991) Evaluation of a linear model of directional selectivity in simple cells of the cat's striate cortex. Vis Neurosci 6:421-428.

Wightman FL, Kistler DJ (1989a) Headphone simulation of free-field listening. I: Stimulus synthesis. J Acoust Soc Am 85:858-867.

Wightman FL, Kistler DJ (1989b) Headphone simulation of free-field listening. II: Psychophysical validation. J Acoust Soc Am 85:868-878.

Wightman FL, Kistler DJ, Perkins ME (1987) A new approach to the study of human sound localization. In: Directional hearing (Yost WA, Gourevitch G, eds), pp 27-48. New York: Springer.

Yost WA, Hafter ER (1987) Lateralization. In: Directional hearing (Yost WA, Gourevitch G, eds), pp 49-84. New York: Springer. 